\title{
The transcriptome of the mosquito Aedes fluviatilis (Diptera: Culicidae), and transcriptional changes associated with its native Wolbachia infection
}

\author{
E. P. Caragata', F. S. Pais², L. A. Baton', J. B. L. Silva', M. H. F. Sorgine ${ }^{3}$ and L. A. Moreira ${ }^{1 *}$
}

\begin{abstract}
Background: Wolbachia is a bacterial endosymbiont that naturally infects a wide range of insect species, and causes drastic changes to host biology. Stable infections of Wolbachia in mosquitoes can inhibit infection with medically important pathogens such as dengue virus and malaria-causing Plasmodium parasites. However, some native Wolbachia strains can enhance infection with certain pathogens, as is the case for the mosquito Aedes fluviatilis, where infection with Plasmodium gallinaceum is enhanced by the native wFlu Wolbachia strain. To better understand the biological interactions between mosquitoes and native Wolbachia infections, and to investigate the process of pathogen enhancement, we used RNA-Seq to generate the transcriptome of Ae. fluviatilis with and without Wolbachia infection.
\end{abstract}

Results: In total, we generated 22,280,160 Illumina paired-end reads from Wolbachia-infected and uninfected mosquitoes, and used these to make a de novo transcriptome assembly, resulting in 58,013 contigs with a median sequence length of $443 \mathrm{bp}$ and an N50 of $2454 \mathrm{bp}$. Contigs were annotated through local alignments using BlastX, and associated with both gene ontology and KEGG orthology terms. Through baySeq, we identified 159 contigs that were significantly upregulated due to Wolbachia infection, and 98 that were downregulated. Critically, we saw no changes to Toll or IMD immune gene transcription, but did see evidence that wFlu infection altered the expression of several bacterial recognition genes, and immune-related genes that could influence Plasmodium infection. wFlu infection also had a widespread effect on a number of host physiological processes including protein, carbohydrate and lipid metabolism, and oxidative stress. We then compared our data set with transcriptomic data for other Wolbachia infections in Aedes aegypti, and identified a core set of 15 gene groups associated with Wolbachia infection in mosquitoes.

Conclusions: While the scale of transcriptional changes associated with wFlu infection might be small, the scope is rather large, which confirms that native Wolbachia infections maintain intricate molecular relationships with their mosquito hosts even after lengthy periods of co-evolution. We have also identified several potential means through which wFlu infection might influence Plasmodium infection in Ae. fluviatilis, and these genes should form the basis of future investigation into the enhancement of Plasmodium by Wolbachia.

Keywords: Aedes fluviatilis, Wolbachia, Transcriptome, RNA-Seq, Metabolism, Mosquito, Vector control, Symbiont, Oxidative stress

\footnotetext{
* Correspondence: luciano@cpqrr.fiocruz.br

${ }^{1}$ Grupo Mosquitos Vetores: Endossimbiontes e Interação Patógeno Vetor,

Centro de Pesquisas René Rachou - Fiocruz, Belo Horizonte, Minas Gerais,

Brazil

Full list of author information is available at the end of the article
} 


\section{Background}

Wolbachia pipientis is a maternally inherited, bacterial endosymbiont that naturally infects an estimated $40 \%$ of terrestrial insect species [1]. Wolbachia induce a wide range of physiological manipulations in different insect hosts, with manipulation of reproductive biology promoting maternal transmission and thus bacterial propagation $[2,3]$. It is through this ability to alter host biology that Wolbachia have gained interest as a form of biological control for the mosquito-transmitted pathogens that are responsible for diseases such as malaria, dengue fever, chikungunya and Zika fever, which represent a serious threat to human health across the globe $[4,5]$.

Many Wolbachia strains induce the reproductive manipulation cytoplasmic incompatibility (CI) in their hosts. This occurs when Wolbachia-infected male insects mate with uninfected females, which then produce unviable eggs. In contrast, Wolbachia-infected females successfully produce viable progeny after mating with either infected or uninfected males [3]. CI increases the proportion of Wolbachia-infected insects over subsequent generations, and serves to replace Wolbachia-uninfected individuals in population a population with those infected by the bacterium [6, 7]. CI-causing strains can be used to suppress mosquito populations that are uninfected by Wolbachia through the release of infected males, similar to the sterile insect technique, or to control Wolbachiainfected populations by releasing mosquitoes infected with a different strain, as this also crashes the population [8].

Several Wolbachia strains also produce antipathogenic effects in their hosts through the pathogen interference phenotype. The mechanics, scope and effectiveness of pathogen interference vary significantly between Wolbachia strains and insect hosts [9-12]. More effective pathogen interference severely inhibits pathogen development and transmission within the host. In Aedes aegypti, a prominent mosquito vector of human diseases, artificial Wolbachia infection interferes with the dengue, Zika, chikungunya, yellow fever and West Nile viruses, and other pathogens including the avian malaria Plasmodium gallinaceum, pathogenic bacteria, and the filarial nematode Brugia pahangi [13-19]. Recent work has also demonstrated that artificial Wolbachia infection in the malaria vector Anopheles stephensi can interfere with infection by the human malaria parasite Plasmodium falciparum, indicating that pathogen interference has broad applicability against human pathogens transmitted by mosquitoes [20].

CI and pathogen interference are the basis for the population replacement form of mosquito control utilised by the Eliminate Dengue Project (http://www.eliminatedengue. com/program). This strategy involves the release of Wolbachia-infected mosquitoes; CI allows the bacterium to spread and become stable within the target, wild population, while pathogen interference makes these mosquitoes less likely to transmit important viruses $[17,21]$. Wolbachia has been successfully spread into a wild Ae. aegypti population [6], with the infection and strong pathogen interference against dengue virus persisting after several years of coevolution [22, 23]

Neither Ae. aegypti nor An. stephensi are known to be naturally infected by Wolbachia. The infections of these mosquitoes described above were generated through transinfection, where Wolbachia is taken from a donor species and then injected into the eggs of the target species to create a stable germline infection transmitted to offspring [20, 24, 25]. In comparison to natural Wolbachia infections, such transinfections typically have a higher bacterial density, and infect a wider range of host tissues, which makes them far more likely to produce pathogen interference, and other extreme manipulations of host physiology [10, 20, 24]. Pathogen interference could potentially be lost from transinfected mosquitoes due to co-evolution between Wolbachia and host, or adaptation on the part of the pathogen [26]. Native Wolbachia infections typically produce minimal pathogen interference, and have little apparent utility to mosquito control strategies that require that trait. However, their low bacterial density, and presumed lower levels of virulence may be reflective the future biological state of transinfected mosquitoes after a long period of adaptation between host and symbiont.

Other native Wolbachia associations can enhance pathogen infection, as is the case for $w$ Pip in Culex pipiens when challenged by Plasmodium relictum [27]. Enhancement is commonly associated with artificial transient somatic Wolbachia infections, and has not been seen with stable germline transinfections [2]. Its mechanism is unknown, but may involve changes to host immunity, metabolism or transcription [27-30]. Needless to say that both loss of pathogen interference, and the development of enhancement would be undesirable consequences if they were to occur in Wolbachia used for vector control. To that end, understanding how native Wolbachia strains influence host physiology at the molecular level will provide useful information about how these strains influence response to pathogens, and potentially highlight a mechanism for enhancement.

The mosquito Aedes fluviatilis inhabits non-urbanized regions throughout Latin America. It is not regarded as a vector for human pathogens in the field, however it is a good laboratory model for $P$. gallinaceum infection [31]. It is naturally infected by the Wolbachia strain $w$ Flu, which grows only to low density, causes CI, and does not induce noticeable fitness costs [15, 32]. The effect of $w$ Flu on dengue virus has not been investigated, however $w$ Flu was shown to enhance $P$. gallinaceum oocyst numbers during some experimental infections [32], making it an interesting model to understand both 
native Wolbachia infections and pathogen enhancement. To determine whether there was a transcriptional basis for this enhancement and to further the understanding of native Wolbachia strains, and the extent to which they impact host biology, we used RNA-Seq to generate the transcriptome of Ae. fluviatilis mosquitoes both with and without their native Wolbachia infection.

\section{Results and Discussion}

\section{RNA sequencing and de novo transcriptome assembly}

We generated a total of 22,280,160 Illumina paired-end reads across 6 Ae. fluviatilis libraries - 3 with Wolbachia infection $(w \mathrm{Flu})$, and 3 where the native infection had been cleared by treatment with tetracycline (Tet). Each library was sequenced from a pool of 16 whole adult female mosquitoes, collected 6 days after eclosion. After the trimming of adaptors and filtering for low quality reads we were left with 19,919,299 high quality paired-end reads $(\mathrm{Q} 30 \%=91)$, across all six libraries. As there was no published genome for Ae. fluviatilis, we attempted to map against the Ae aegypti predicted transcriptome with Bowtie 2 , but less than $25 \%$ could be successfully mapped, which was unsuitable for further analysis. We then used the complete set of reads to make a de novo transcriptome assembly with Trinity (see methods). A total of 58,013 contigs were assembled encompassing 64 million base pairs (bp), with a median sequence length of $443 \mathrm{bp}$ and an N50 of $2454 \mathrm{bp}$ (Table 1). Over 19,000 contigs were larger than $1 \mathrm{~Kb}$ in size.

\section{Functional annotation}

Annotation was performed through local alignments using BlastX (with an $e$-value of 1e-10) against the NCBI nonredundant database (NR), which returned annotation for 26,066 contigs. Of these, 14,209 contained unique hits

Table 1 Summary of sequencing results

\begin{tabular}{|c|c|c|}
\hline \multirow[t]{4}{*}{ Paired-end reads } & Total base pairs & $19,919,299$ \\
\hline & Median length & $171 \times 2$ \\
\hline & Q20 (\%) & 99 \\
\hline & Q30 (\%) & 91 \\
\hline \multirow{6}{*}{$\begin{array}{l}\text { De novo } \\
\text { assembly }\end{array}$} & Total contigs & 58,013 \\
\hline & Transdecoder ORFs & 24,329 \\
\hline & Total bp in all contigs & $64,257,972$ \\
\hline & Median contig size (bp) & 443 \\
\hline & Contigs larger than $1 \mathrm{~Kb}$ & 19,414 \\
\hline & N50 & 2454 \\
\hline \multirow{2}{*}{$\begin{array}{l}\text { Annotated } \\
\text { contigs }\end{array}$} & $N R$ & 26,066 \\
\hline & KEGG & 25,312 \\
\hline \multirow[t]{2}{*}{ Mapping } & $\begin{array}{l}\text { Paired reads aligned concordantly } 1 \text { time } \\
\text { (\%) }\end{array}$ & 66.78 \\
\hline & Overall alignment rate (\%) & 95.01 \\
\hline
\end{tabular}

(Table 1). As previous molecular characterization of Ae. fluviatilis has been limited to a few microsatellites [33], our analysis relied entirely on finding similarity to other genomes. Consequently, 31,947 contigs (55\% of the entire data set) did not get a significant BlastX hit, which could be explained by misassembly or from lack of representation in the NR database. 920 of these were considered to be protein-coding sequences based on predictions made by Transdecoder, but no further characterization was possible for the remaining 31,027 contigs. For the purposes of our analysis we have assumed that gene function is identical to what was described in the blast hits, but this may not be the case, as genetic homology does not always imply functional homology. However, we are fairly confident given that majority of annotations were previously described in closely related mosquitoes.

The assembled transcriptome of Ae. fluviatilis showed a high degree of similarity to Ae. aegypti, with 18,082 $(69.38 \%)$ of the annotated contigs most closely matched to that species (Fig. 1). This was not surprising as Ae. aegypti has the most thoroughly annotated genome of the mosquitoes in the Aedes genus, meaning that this observed similarity may have more to do with the prevalence of Ae. aegypti sequences in public databases than the closeness of the relationship between the two species [34]. A further 5,616 (21.55\%) of the annotated contigs were homologous to genes from other mosquito species, and overall $94.9 \%$ of the matches from BlastX were arthropod in origin. We conducted divergence analyses to better clarify the relationship between Ae. fluviatilis and other mosquito species (Additional file 1), and determined that Ae. fluviatilis diverged from these species approximately 98 million years ago ( $95 \%$ highest posterior density interval: 64.1 to 133.5 million years ago). A further 383 contigs matched to non-arthropod animals.

Across both the Tet and $w$ Flu libraries we identified 751 contigs of bacterial origin (2.86\%), and 112 of fungal origin $(0.43 \%)$, which may potentially represent part of the Ae. fluviatilis intestinal microbiota, or could be the result of environmental contamination during sample collection. The bacterial sequences represented 250 distinct taxa, with the majority associated with a single contig. The diversity of sequence origins may indicate that the microbiota comprises many species, or that the majority of gut flora belonged to species that were not represented in the NR database. It is also possible that some of these contigs actually came from Wolbachia, but were different in composition from previously sequenced Wolbachia genes. Given the relatively low number of contigs of bacterial origin, the low copy numbers of these contigs and the low number of hits for each bacterial taxon in our data set, it was not possible to gain significant insight into the potential influence of $w$ Flu on the composition or role of the microbiota. 


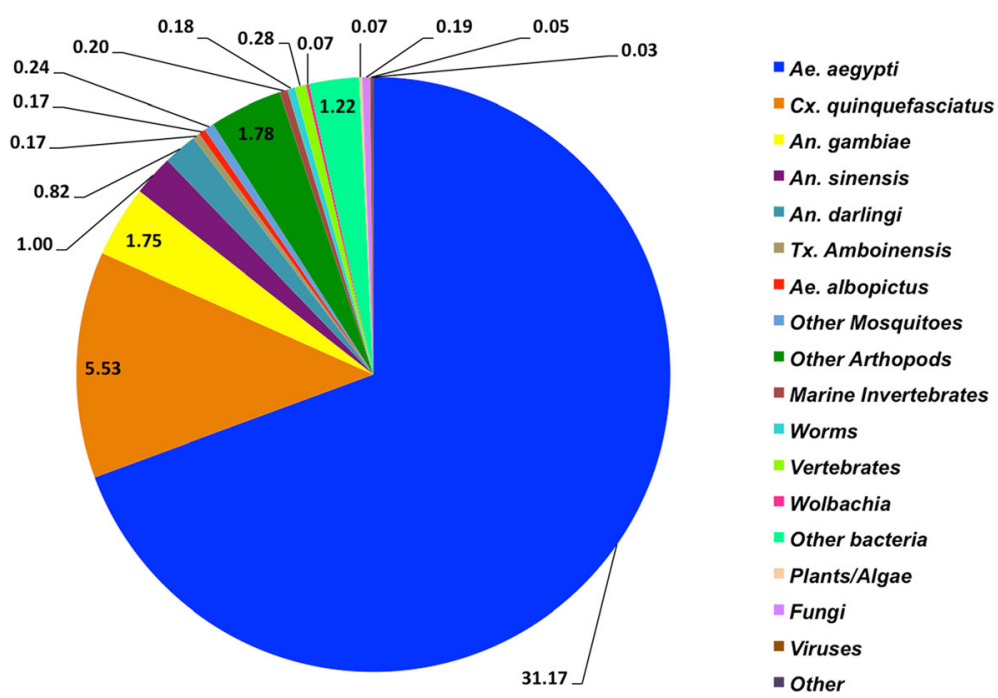

Fig. 1 Breakdown of contig annotations by organism of origin. After de novo assembly, contigs were annotated by local alignment with the BlastX database. The chart depicts the percentage of contigs where the most significant BlastX hit matched to a particular species of clade. 90.93\% of the hits were to a mosquito species. Contigs without a BlastX hit $(31,951)$ are not shown

Only 42 contigs of Wolbachia origin were identified in our data (Additional file 2). These contigs came from a variety of strains including $w \mathrm{Ana}, w \mathrm{Bm}, w \mathrm{MelPop}$, and $w$ Pip. 38 were expressed in the $w$ Flu libraries, and 4 in the Tet libraries - potentially via integration into the host genome. Wolbachia genomes typically contain between 900-1400 coding sequences [35]. Of the 42 identified Wolbachia contigs, 22 were hypothetical proteins with unknown function. Interestingly, one contig was a heme biosynthesis protein, which suggests that $w$ Flu promotes heme production, and provides further evidence to support the theory that Wolbachia alters host iron and redox homeostasis, even amongst native associations [36, 37]. We also identified a glycosidase hydrolase, which plays a role in sugar metabolism. The remainder of the Wolbachia contigs fell into two broad functional categories. The first included genes involved in DNA/RNA processing, DNA repair and RNA synthesis, and are likely part of normal Wolbachia replication and transcription processes, as similar genes have been identified in the $w \mathrm{Mel}$ genome [38]. A further group of contigs included Wolbachia membrane proteins, and ankyrin genes, which are used to attach the bacterial membrane to the host cytoskeleton. These genes likely represent part of the machinery used to mediate Wolbachia-host interactions [39], are typically present in large numbers in Wolbachia genomes [35, 38, 40-42], and may also be involved in CI [43].

The genomes of both Wolbachia strains and their insect hosts typically contain large quantities of mobile elements including transposons [38]. These can alter or disrupt the expression of genes, depending on their point of insertion into the host genome [34, 44]. We found 61 contigs related to transposable elements, none of which were matched to a Wolbachia genome. We also found a further 28 of viral origin, which could represent sequences from past or present members of the Ae. fluviatilis viral flora, which have not been well characterised.

As part of the contig annotation process, gene ontology (GO) and KEGG orthology (KO) terms for each contig were identified using Blast2GO and the KEGG database, respectively (Fig. 2). We identified genes associated with a wide range of biological processes, functions and structures. From the whole set of contigs, 23,035 were assigned to at least one GO term. The majority of contigs in the biological process category were associated with cellular or metabolic process. Cell, and cell part were the cellular component terms with the greatest frequency, while binding and catalytic activity were the most common terms associated with the molecular function category. The most common $\mathrm{KO}$ terms were related to diseases, and molecular information processing.

\section{Differentially expressed contigs}

Each RNA-Seq library was independently mapped to the assembled transcriptome using Bowtie. An average of $95 \%$ of the reads from each library were successfully mapped, and these data were used to generate counts for each contig. A list of differentially expressed contigs was generated using the baySeq package from Bioconductor, with $66 \%$ of these contigs (data not shown) also determined to be differentially expressed via analysis with the DESeq2 package, again from Bioconductor. Given this high level of concordance between the lists, we chose to proceed with further analysis of the baySeq 


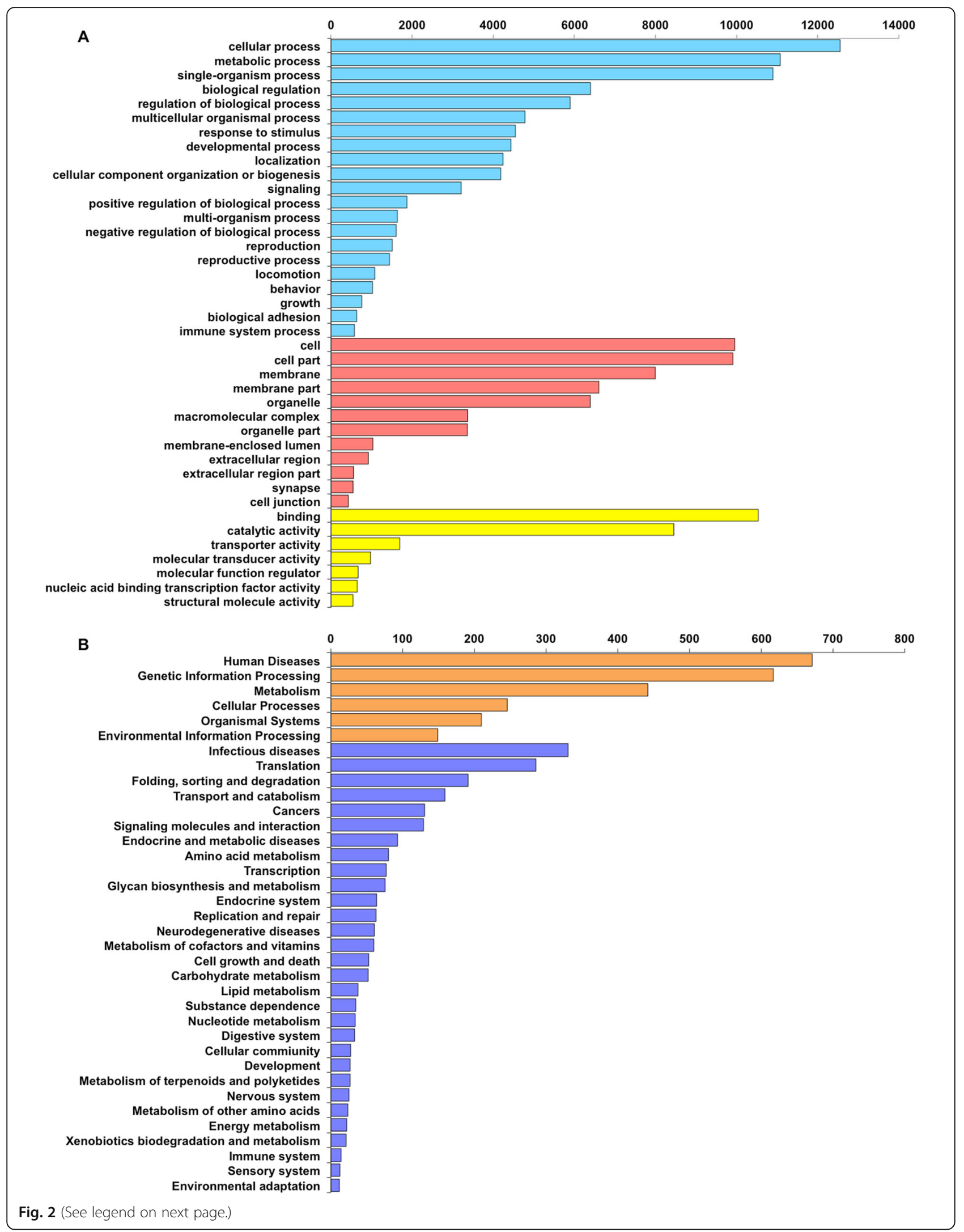


(See figure on previous page.)

Fig. $2 \mathrm{GO}$ and $\mathrm{KO}$ terms associated with the Aedes fluviatilis transcriptome. a The 40 most enriched gene ontology terms in the biological process (blue), cell component (red) and molecular function (yellow) categories at level 2. b First level (orange) and second level (purple) KEGG orthology functional category terms associated with Ae. fluviatilis. GO and KO term lists were generated using combined data for both the Wolbachia-infected and -uninfected libraries

list, as that method of analysis is known to be more sensitive [45]. Through baySeq we calculated the FPKM (Fragments Per Kilobase of transcript per Million mapped reads) for each contig, which considers the size of the contig in base pairs, and the overall data set size, as a measure of expression.

A total of 257 differentially expressed contigs were identified using baySeq, 159 were associated with the $w$ Flu libraries, and 98 with the Tet libraries. Of these, 50 (19.4\%) from the $w$ Flu libraries (upregulated by $w$ Flu) and $32(32.7 \%)$ from the Tet libraries (downregulated by $w$ Flu) had no matches after the BlastX search. This left
109 and 66 possible orthologs to known genes with increased and decreased expression due to Wolbachia infection, respectively (Additional file 3). Excluding multiple matches to genes with the same accession number, there were 95 unique contigs that were upregulated by Wolbachia, and 59 unique contigs that were downregulated, on the whole mosquito level. It is possible that analysis of whole mosquitoes precluded detection of genes that experienced a pattern of tissue-specific differential expression.

To confirm the accuracy of the sequencing data, the expression of six contigs was examined using RT-qPCR (Fig. 3). Two contigs, comp10645_c1_seq2 and comp10453_c0_seq5
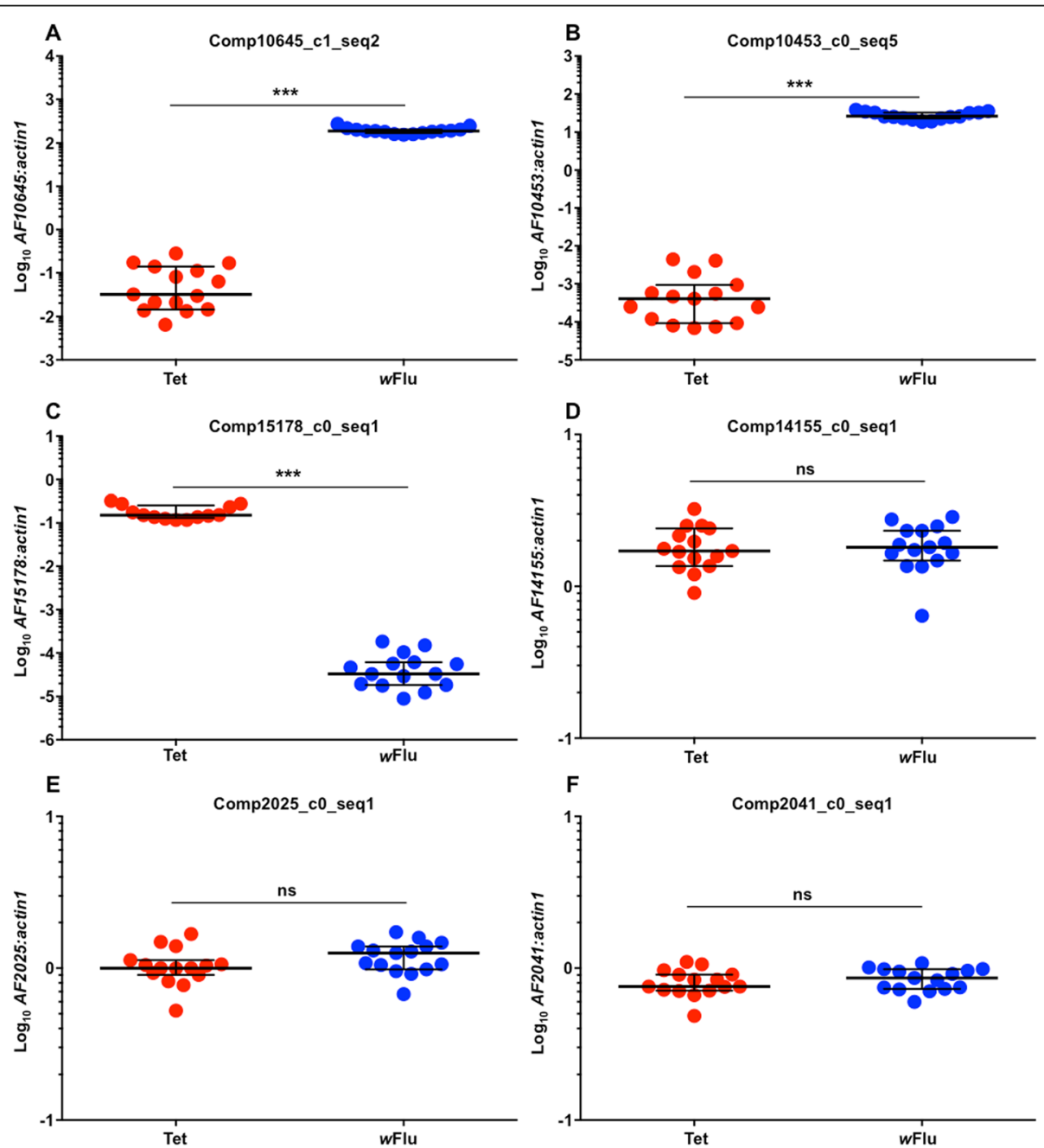

Fig. 3 Confirmation of contig differential expression using RT-qPCR. 4 differentially expressed contigs (a-d), and 2 non-differentially expressed contigs (e-f) were selected at random, and expression levels were quantified via RT-qPCR. 5/6 contigs performed as expected, while one that was expected to show higher expression in Wolbachia-infected mosquitoes did not 
were predicted to be upregulated by $w F l u$, and further analysis confirmed that expression was significantly higher in $w$ Flu mosquitoes (Mann Whitney U tests: $10645-P<$ $0.0001 ; 10453-P<0.0001)$. A further 2 contigs were predicted to be downregulated by $w$ Flu, and the first of these, comp15178_c0_seq1, demonstrated that pattern (Mann Whitney U test: $P<0.0001$ ). However for the second, comp14155_c0_seq1, expression levels were not significantly different due to the presence of Wolbachia (Unpaired t test: $t=0.1196 P=0.9057$ ). All four of those contigs were predicted to display differential expression in both the bayseq and DESeq2 analyses. Only low levels of comp14155_c0_seq1 were detected during sequencing, which could explain the lack of differential expression observed during RT-qPCR. The final 2 contigs that were examined were predicted to have equivalent expression levels between treatments, and both fit that pattern (Unpaired t tests: comp2025_c0_seq1 $-t=1.500, P=$ 0.1449; comp2041_c0_seq1 $-t=0.7994, P=0.4308$ ). Further validation of the differential expression of these genes using different techniques will likely prove valuable for future research.

$\mathrm{GO}$ information for the annotated contigs was retrieved from Blast2GO, or repositories of transcriptional data such as FlyBase and VectorBase for some genes where Blast2GO produced no information (see methods). GO information could not be found for 28 upregulated contigs, and 17 downregulated contigs. The remaining upregulated contigs were associated with 286 GO terms, of which 93 terms had multiple hits. While downregulated contigs were associated with $190 \mathrm{GO}$ terms, and $62 \mathrm{GO}$ had multiple hits (Additional file 4). These GO terms and GenBank annotations were used to group the differentially expressed contigs based on their putative functions, and this information was used to develop profiles of the transcriptional changes that occurred both with (Table 2) and without (Table 3) wFlu infection. Some contigs had more than one annotated function, and are listed in multiple categories.

\section{Immune stimulation and suppression}

Transinfection with Wolbachia in mosquito species has typically led to widespread increases in the expression of immune genes, including those involved in the Toll and IMD immune pathways, and a large number of antimicrobial peptides $[10,11,14,20]$. This immune activation has been linked to interference of dengue viruses in mosquitoes [10, 14, 15], and immune suppression has been hypothesized as a potential cause of the enhancement of Plasmodium infection seen in some native and transient Wolbachia infections [28, 29]. We observed no changes in the transcription of genes in the Toll or IMD pathways, including genes such as rel1, or rel2, which might have been indicative of a change in pathway regulation. We also saw no changes in the expression of any of the antimicrobial peptides commonly affected by Wolbachia. The absence of systemic immune activation is common amongst native Wolbachia infections, and may be symptomatic of increased tolerance on the part of the host, and reduced pathogenicity on the part of the symbiont $[46,47]$.

We did observe differential expression of 6 contigs directly involved in mosquito immunity, 4 upregulated ( 2 cell wall hydrolases, a galactose specific c-type lectin, and a gram negative bacteria binding protein) and 2 downregulated (a mucin like protein, and a galactose specific c-type lectin). These genes are typically associated with bacterial binding and degradation, but many have also been linked to Plasmodium infection and could have contributed to the enhancement of P. gallinaceum infection in Ae. fluviatilis [32]. Gram negative bacteria binding proteins, for instance can have a broader role in immune stimulation, and are involved in the response to Plasmodium infection in An. gambiae [48]. Some galactose-specific c-type lectins are highly differentially expressed by Wolbachia [11], their expression is stimulated by Plasmodium infection, and they have been shown to protect Plasmodium against melanisation by the host immune system [49]. There are also examples of mucin like genes and cell wall hydrolases that are critical to Plasmodium development in mosquitoes [50, 51].

We also identified other differentially expressed genes have also been linked with insect immunity or Plasmodium infection. Plasmodium infection is dependent on glycolysis, and the metabolism of amino acids and lipids [52], all affected by $w$ Flu infection (see below). There was evidence of altered sugar metabolism and transport, which could promote the development of Plasmodium [53]. Additionally, there was increased expression of a $\beta$-hexosaminidase, and these genes have a peptidoglycan hydrolase effect, can be bactericidal, and have a broader immune function that could facilitate interaction with Plasmodium [54]. Finally, we also saw upregulation and downregulation of serine proteases, which are involved in both digestion and immunity. Serine proteases have been linked to Plasmodium growth and development, invasion of host cells, and can protect the parasite against the host oxidative stress response [55, 56]. Any of these transcriptional changes could be linked to the enhancement of $P$. gallinaceum, and therefore merit further investigation.

\section{Redox process}

One interesting characteristic of Wolbachia infection is the breakdown of redox homeostasis, which has been seen across multiple infected hosts $[57,58]$. This effect often manifests through the induction of reactive oxygen 
Table 2 Significantly upregulated contigs

\begin{tabular}{|c|c|c|c|c|}
\hline Component number & Name & RPKM - wFlu & RPKM - Tet & Function \\
\hline \multicolumn{5}{|c|}{ 1. Membranes \& membrane transport } \\
\hline comp7819_c0_seq1 & Niemann-pick type c2 & 268.16 & 88.12 & Cholesterol transport \\
\hline comp10576_c0_seq2 & UNC93a protein & 74.60 & 43.64 & Transmembrane transport \\
\hline comp13082_c0_seq1 & Membrane transport protein, putative & 52.03 & 16.70 & Transmembrane transport \\
\hline comp5926_c0_seq2 & Monocarboxylate transporter & 28.09 & 14.45 & Transmembrane transport \\
\hline comp9373_c0_seq2 & CD36 antigen & 12.48 & 5.80 & Cell adhesion/lipid binding \\
\hline comp6262_c1_seq3 & Gustatory receptor 64a & 9.64 & 2.24 & Chemosensory receptor \\
\hline comp9362_c0_seq1 & Ionotropic receptor 100a & 9.14 & 2.02 & Chemosensory receptor \\
\hline comp14845_c0_seq1 & OMPA-like protein & 5.99 & 0.00 & Wolbachia membrane \\
\hline comp13777_c0_seq18 & Permease & 5.26 & 0.00 & Transmembrane transport \\
\hline comp6505_c0_seq1 & Glucose transport protein & 2.15 & 0.22 & Glucose transport \\
\hline comp6457_c0_seq3 & Sulfakinin receptor & 1.19 & 0.25 & Chemosensory receptor \\
\hline \multicolumn{5}{|l|}{ 2. Redox response } \\
\hline comp13612_c0_seq1 & Chorion peroxidase & 287.11 & 168.42 & Oxidative stress \\
\hline comp8621_c0_seq1 & Anterior fat body protein & 135.03 & 79.59 & Redox process \\
\hline comp11151_c0_seq1 & Cytochrome p450 & 81.07 & 29.11 & Oxidase \\
\hline comp8229_c0_seq2 & Cytochrome p450 & 50.12 & 26.71 & Oxidase \\
\hline comp10103_c0_seq2 & $\begin{array}{l}\text { Neuferricin homolog, cytochrome } \\
\text { b5 domain-containing protein } 2\end{array}$ & 25.33 & 12.90 & Heme binding \\
\hline comp4282_c0_seq1 & Cytochrome p450 & 9.14 & 3.10 & Oxidase \\
\hline comp6824_c0_seq1 & Short-chain dehydrogenase & 6.79 & 1.43 & Oxidoreductase \\
\hline \multicolumn{5}{|l|}{ 3. Metabolism } \\
\hline comp10525_c0_seq3 & Brain chitinase and chia & 196.30 & 85.85 & Amino acid/carbohydrate metabolism \\
\hline comp7163_c0_seq1 & Chymotrypsin 1 & 179.35 & 69.13 & Proteolysis \\
\hline comp11118_c0_seq1 & Gram negative bacteria binding protein 2 & 149.39 & 79.07 & Carbohydrate metabolism \\
\hline comp8621_c0_seq1 & Anterior fat body protein & 135.03 & 79.59 & Lipoprotein \\
\hline comp9990_c0_seq1 & Phosphatidylcholine-sterol acyltransferase & 87.08 & 32.92 & Sterol/phospholipid metabolism \\
\hline comp2787_c0_seq1 & Serine protease & 56.13 & 24.71 & Proteolysis \\
\hline comp4890_c0_seq1 & Trypsin $\theta$ & 47.87 & 12.96 & Proteolysis \\
\hline comp12424_c0_seq4 & Pancreatic triacylglycerol lipase & 45.47 & 19.55 & Fat metabolism \\
\hline comp10453_c0_seq5 & Orf16-lacz fusion protein & 42.79 & 0.88 & Carbohydrate/glycoprotein metabolism \\
\hline comp12444_c0_seq3 & Glucosyl/glucuronosyl transferases & 37.48 & 12.40 & Carbohydrate/fatty acid metabolism \\
\hline comp10786_c0_seq2 & Serine protease & 36.87 & 17.84 & Proteolysis \\
\hline comp13425_c0_seq4 & Chitin metabolism protein & 33.94 & 10.39 & Peritrophic membrane metabolism \\
\hline comp11198_c1_seq2 & Serine 3-dehydrogenase & 28.11 & 13.52 & Amino acid metabolism \\
\hline comp8292_c0_seq2 & Galactose-specific C-type lectin & 26.69 & 2.38 & Carbohydrate metabolism \\
\hline comp9458_c0_seq1 & a chain crystal structure of $\beta$-glucosidase & 18.76 & 5.39 & Carbohydrate metabolism \\
\hline comp12611_c0_seq1 & $\beta$-hexosaminidase B & 17.00 & 5.71 & Carbohydrate/aminosugar metabolism \\
\hline comp9289_c0_seq1 & Serine threonine-protein kinase RIO1 & 16.31 & 3.07 & Proteolysis \\
\hline comp13777_c0_seq6 & PG1 homology to homo sapiens & 14.47 & 0.55 & Organic compound metabolism \\
\hline comp12709_c0_seq1 & Y glutamyl transpeptidases & 8.10 & 3.80 & Amino acid metabolism \\
\hline comp5863_c0_seq3 & Acyltransferase & 5.78 & 0.76 & Protein modification \\
\hline comp12058_c1_seq1 & O-linked n-acetyl glucosamine transferase & 5.75 & 0.27 & Aminosugar metabolism \\
\hline comp6912_c0_seq1 & Glucosyl/glucuronosyl transferases & 2.70 & 0.57 & Carbohydrate/fatty acid metabolism \\
\hline
\end{tabular}


Table 2 Significantly upregulated contigs (Continued)

\begin{tabular}{|c|c|c|c|c|}
\hline \multicolumn{5}{|l|}{ 4. Signalling } \\
\hline comp9906_c0_seq2 & CDC42 protein & 118.57 & 58.48 & GTPase \\
\hline comp8210_c0_seq1 & Odorant-binding protein a10 & 49.07 & 17.86 & Chemosensory protein serine/threonine kinase \\
\hline comp8312_c0_seq6 & GTP-binding protein di-ras2-like & 24.21 & 13.00 & GTPase \\
\hline comp10042_c0_seq3 & Stretchin- isoform $v$ & 5.39 & 0.27 & Protein kinase \\
\hline comp309_c0_seq1 & $\rho$ guanyl-nucleotide exchange factor & 3.48 & 0.49 & GTPase \\
\hline \multicolumn{5}{|l|}{ 5. Cell Process } \\
\hline comp7767_c0_seq1 & Protein frg1 homolog & 69.85 & 39.25 & rRNA processing/Gene silencing/activation \\
\hline comp13326_c1_seq7 & Histone h2b & 49.34 & 27.23 & Gene silencing/activation \\
\hline comp10542_c0_seq1 & Integrator complex subunit 12 & 27.74 & 10.71 & snRNA processing \\
\hline comp6248_c0_seq1 & Chromobox protein homolog 1 & 20.33 & 7.35 & Gene silencing/activation \\
\hline comp13777_c0_seq25 & Ribosomal protein s10 & 13.45 & 3.44 & Protein synthesis \\
\hline comp7336_c0_seq1 & Hect e3 ubiquitin ligase & 11.86 & 5.97 & DNA repair \\
\hline comp5798_c0_seq1 & Zinc finger protein 425 & 10.96 & 2.44 & Transcription factor \\
\hline comp7025_c0_seq1 & Muts protein homolog 5-like & 10.24 & 5.38 & DNA repair \\
\hline comp8754_c0_seq1 & Mitochondrial 28 s ribosomal protein s29 & 8.11 & 0.00 & Translation \\
\hline comp15539_c0_seq1 & $\begin{array}{l}\text { TcasGA2_TC002223 Zinc finger domain containing } \\
\text { protein }\end{array}$ & 4.07 & 0.00 & Transcription factor \\
\hline comp11749_c0_seq1 & Muts protein homolog 4 & 3.58 & 1.18 & DNA repair \\
\hline comp2214_c0_seq1 & RNase $\mathrm{H}$ and integrase-like protein & 3.60 & 0.74 & DNA replication \& repair \\
\hline comp13817_c0_seq21 & RNA/mRNA processing protein & 2.14 & 0.14 & RNA/mRNA processing \\
\hline \multicolumn{5}{|l|}{ 6. Physiology } \\
\hline comp13612_c0_seq1 & Chorion peroxidase & 287.11 & 168.42 & Ovarian follicle maturation \\
\hline comp5400_c0_seq1 & Synaptic vesicle protein & 37.67 & 20.32 & Neurotransmitter trafficker \\
\hline comp13425_c0_seq4 & Chitin metabolism protein & 33.94 & 10.39 & Morphogenesis \\
\hline comp11035_c0_seq1 & Voltage-dependent para-like sodium channel & 27.45 & 7.89 & Nerve impulse \\
\hline comp7184_c0_seq1 & Fasciculation and elongation protein $\zeta-2$ & 22.96 & 6.83 & Signal transduction/neural development \\
\hline comp14297_c0_seq1 & Major allergen bla g & 22.81 & 5.58 & Neurotransmitter binding \\
\hline comp11161_c0_seq1 & Neuroblast formation protein & 15.44 & 3.44 & Cell division/neuroblast formation \\
\hline comp11781_c0_seq19 & BMP-induced, dendrite morphogenesis factor & 2.75 & 1.08 & Neural development \\
\hline comp17984_c0_seq1 & Voltage-dependent pq type calcium channel & 2.36 & 0.00 & Neurotransmitter release \\
\hline \multicolumn{5}{|l|}{ 7. Immunity } \\
\hline comp11118_c0_seq1 & Gram negative bacteria binding protein 2 & 149.39 & 79.07 & Pathogen binding \\
\hline comp10645_c1_seq8 & Cell wall-associated hydrolase & 42.78 & 5.99 & Bacterial cell wall degradation \\
\hline comp8292_c0_seq2 & Galactose-specific C-type lectin & 26.69 & 2.38 & Pathogen binding \\
\hline comp14485_c0_seq1 & Cell wall-associated hydrolase & 12.27 & 1.61 & Bacterial cell wall degradation \\
\hline \multicolumn{5}{|l|}{ 8. Salivary Proteins } \\
\hline comp4837_c0_seq1 & Salivary basic peptide-1 & 259.44 & 69.40 & Salivary protein \\
\hline comp5901_c0_seq1 & Anti-platelet protein & 66.88 & 39.76 & Salivary protein \\
\hline \multicolumn{5}{|l|}{ 9. Mobile Elements } \\
\hline comp6660_c0_seq1 & af378002_1 transposase & 20.28 & 8.02 & DNA integration \\
\hline comp13085_c0_seq2 & Reverse transcriptase & 10.99 & 5.13 & Reverse transcriptase \\
\hline comp12175_c0_seq4 & Retrovirus transposon polyprotein & 7.68 & 3.39 & Polyprotein \\
\hline comp13891_c0_seq14 & af378002_1 transposase & 7.41 & 0.00 & DNA integration \\
\hline
\end{tabular}


Table 2 Significantly upregulated contigs (Continued)

\begin{tabular}{lllll}
\hline comp14791_c0_seq1 & Uncharacterized protein K02A2 & 6.41 & 0.15 & DNA integration \\
comp14916_c0_seq1 & Retrovirus transposon polyprotein & 4.76 & 0.00 & Polyprotein \\
comp13573_c0_seq26 & Endonuclease-reverse transcriptase & 1.20 & 0.10 & Reverse transcriptase \\
\hline
\end{tabular}

Contigs in italics are of bacterial origin. FPKM - Fragments Per Kilobase of transcript per Million mapped reads for the wFlu and Tet libraries

species (ROS), and altered expression of genes involved in oxidative stress response, which occur with both native Wolbachia associations and transinfections [10, 12, 58], but it is unclear if they represent an immune response to Wolbachia, or normalization of the redox processes altered by infection [37]. Increased oxidative stress has been linked to pathogen interference against viruses and Plasmodium [10, 11, 20], and may be part of Wolbachia's immune evasion strategy [37]. Similarly, higher oxidative stress levels are an important part of the anti-Plasmodium immune response [59].

In Ae. fluviatilis we saw evidence of an altered redox response due to $w$ Flu infection, in the form of higher levels of a short-chain dehydrogenase, an oxidoreductase, which can induce ROS, and 3 cytochromes $\mathrm{p} 450$, which act as oxidases $[60,61]$. Wolbachia have also been linked with iron metabolism and storage, and this can influence key physiological traits such as fecundity [62-64]. wFlu infection induced higher levels of neuferricin, a protein that binds iron-rich heme, which is interesting given that Wolbachia produce enzymes involved in heme synthesis [38], and may utilise it as an energy source [36]. Likewise, heme is essential to the development of Plasmodium in mosquitoes [65]. Given that $w$ Flu appears to alter redox homeostasis, it would be interesting to see if $w$ Flu infection induces higher ROS levels. It should be noted that tetracycline treatment impacts mitochondrial function, which can lead to changes in insect oxidative stress response [66]. While we did use tetracycline to clear the $w$ Flu infection, our experiments were performed more than 2 years (approximately 30 generations) after antibiotic treatment.

\section{Metabolism}

We observed that 33 genes associated with metabolism and digestion were differentially expressed as a result of $w$ Flu infection. Wolbachia demonstrate many nutritionbased physiological changes in different insect hosts, including mutualism through nutritional provision [40, 67], better performance under nutritional stress [62], and competition for nutrients leading to effects on host fecundity and fertility, and on pathogen interference $[68,69]$. These processes are linked to a variety of nutrients and micronutrients including amino acids, iron, and flavin adenine dinucleotide, which indicates that Wolbachia interact with a wide range of host metabolic pathways.

Wolbachia infections in Ae. aegypti show differential expression of large numbers of digestive enzymes, particularly serine proteases and trypsins, which may indicate cannibalisation of host resources to promote Wolbachia propagation. Infection with $w$ Flu increased the expression of 3 serine proteases, and 2 trypsins, and decreased levels of two other serine proteases and 2 zinc metallopeptidases. While the number of affected genes were fewer than seen for the Ae. aegypti transinfections it is sufficient to suggest that a similar process operates in Ae. fluviatilis. Likewise, $w$ Flu infection elevated levels of brain chitinase and chia, an enzyme involved in breakdown of the peritrophic matrix, which may be further evidence of an altered digestive process, and could compromise the integrity of the peritrophic matrix and potentially facilitate Plasmodium invasion.

We observed that $w$ Flu infection had a broad effect on several aspects of host metabolism, including carbohydrate and lipid metabolism, both areas where Wolbachia is lacking key biosynthesis genes, and where Wolbachia transinfection alters transcription in Ae aegypti [11, 38, 70]. Previous work indicates that $w$ Flu infection leads to elevated levels of glycogen [71], a major carbohydrate reserve, in developing embryos. While it is unclear if this effect occurs in adults, we did see elevated levels of $\beta$ glucosidase, which encodes an enzyme involved in the breakdown of complex carbohydrates including glycogen. Similarly, $\beta$-hexosaminidase $b$ levels were also higher, with this enzyme involved in the hydrolysis of amino-sugars. Wolbachia heavily utilise host lipids and sterols, and alter the cellular lipid profile $[68,72,73]$, potentially to serve bacterial propagation [74], with similar processes underlying Plasmodium development [75]. Lipid metabolism genes affected by $w$ Flu had a diverse range of functions and include glucosyl glucuronosyl transferases, enzymes required for glucuronidation, which has a role in the metabolism of fatty acids and other compounds, and also pancreatic triacylglycerol lipases, which are used to digest triacylglycerides and process fats. Interestingly, $w$ Flu also increased levels of phosphatidylcholine-sterol acyltransferase, which is involved in sterol and phospholipid metabolism, and may 
Table 3 Significantly downregulated contigs

\begin{tabular}{|c|c|c|c|c|}
\hline Component number & Name & RPKM - wFlu & RPKM - Tet & Function \\
\hline \multicolumn{5}{|c|}{ 1. Membranes \& membrane transport } \\
\hline comp7683_c0_seq2 & Membrane glycoprotein lig-1 & 7.27 & 22.40 & Membrane receptor \\
\hline comp3093_c0_seq1 & EGF-like module-containing mucin-like hormone receptor-like 1 & 2.09 & 11.49 & Membrane receptor \\
\hline comp1729_c0_seq1 & $A B C$ transporter ATP-binding protein & 0.33 & 4.41 & Transmembrane transport \\
\hline \multicolumn{5}{|l|}{ 2. Redox response } \\
\hline comp5605_c0_seq1 & Anterior fat body protein & 16.34 & 36.66 & Redox process \\
\hline comp8781_c0_seq2 & Dimethylaniline monooxygenase & 0.42 & 2.93 & Redox process \\
\hline \multicolumn{5}{|l|}{ 3. Metabolism } \\
\hline comp5123_c0_seq1 & Serine protease 14 & 85.21 & 201.81 & Proteolysis \\
\hline comp8292_c1_seq1 & Galactose-specific C-type & 66.95 & 145.97 & Carbohydrate metabolism \\
\hline comp10957_c0_seq1 & Serine protease & 47.69 & 111.75 & Proteolysis \\
\hline comp8332_c0_seq1 & Diacylglyceride synthesis protein & 20.73 & 48.52 & Fat synthesis \\
\hline comp5605_c0_seq1 & Anterior fat body protein & 16.34 & 36.66 & Lipoprotein \\
\hline comp7785_c0_seq8 & Glucosamine 6-phosphate N-acetyltransferase, putative & 10.17 & 23.93 & Amino acid/aminosugar metabolism \\
\hline comp14516_c0_seq1 & Zinc metalloprotease & 0.71 & 12.68 & Proteolysis \\
\hline comp9411_c0_seq5 & Carboxylesterase & 1.78 & 7.61 & Hydrolase \\
\hline comp2544_c0_seq1 & Vitellogenic carboxypeptidase & 0.80 & 6.73 & Proteolysis \\
\hline comp16467_c0_seq1 & Zinc metallopeptidase & 0.00 & 4.09 & Proteolysis \\
\hline comp17222_c0_seq1 & Acyltransferase & 0.25 & 3.39 & Protein modification \\
\hline \multicolumn{5}{|l|}{ 4. Signalling } \\
\hline comp9906_c0_seq1 & CDC42 protein & 11.93 & 69.66 & GTPase \\
\hline comp11677_c0_seq3 & Dual specificity tyrosine-phosphorylation-regulated kinase & 13.96 & 31.43 & TPR Kinase \\
\hline comp7548_c0_seq2 & Calmodulin-like protein 4-like & 13.13 & 30.04 & Signal transduction \\
\hline comp5282_c0_seq1 & Signal peptidase complex subunit 1 & 5.76 & 26.11 & Signal protein cleavage \\
\hline comp11677_c0_seq2 & Dual specificity tyrosine-phosphorylation-regulated kinase & 13.17 & 25.49 & TPR Kinase \\
\hline comp10289_c0_seq6 & Guanine nucleotide-binding protein subunit & 5.47 & 16.12 & Signal protein \\
\hline comp9283_c0_seq2 & GTP-binding protein alpha gna & 1.08 & 4.54 & GTPase \\
\hline \multicolumn{5}{|l|}{ 5. Cell Process } \\
\hline comp14155_c0_seq1 & T-complex polypeptide 20 & 0.94 & 25.10 & Protein folding \\
\hline comp8487_c0_seq1 & Histone h1 & 6.28 & 18.53 & DNA binding \\
\hline comp4291_c0_seq2 & RNase $\mathrm{h}$ and integrase-like protein & 3.25 & 15.80 & DNA repair \\
\hline comp6475_c0_seq2 & Insulin receptor tyrosine kinase substrate & 0.21 & 3.09 & Cytoskeleton organization \\
\hline \multicolumn{5}{|l|}{ 6. Physiology } \\
\hline comp13996_c0_seq1 & Rhodopsin receptor 1 & 3383.34 & 7140.82 & Visual receptor \\
\hline comp9690_c0_seq8 & Photoreceptor protein & 1087.17 & 2438.80 & Phototransduction \\
\hline comp8001_c0_seq1 & Ultraviolet-sensitive opsin & 78.20 & 207.73 & Visual receptor \\
\hline comp12700_c0_seq1 & Calmodulin-binding protein trpl & 41.91 & 101.60 & Phototransduction \\
\hline comp9621_c0_seq1 & Blue-sensitive visual pigment & 16.68 & 56.19 & Phototransduction \\
\hline comp3173_c0_seq1 & Rhodopsin & 19.70 & 51.63 & Visual receptor \\
\hline comp2653_c0_seq1 & Mucin-like peritrophin & 11.81 & 39.14 & Petritrophic matrix component \\
\hline comp8706_c0_seq1 & Cuticular protein isoform a & 1.62 & 10.60 & Cuticle component \\
\hline comp4000_c0_seq1 & Photoreceptor protein & 0.00 & 8.21 & Phototransduction \\
\hline comp5558_c0_seq1 & Hypothetical protein ZHAS & 0.43 & 3.63 & Cell growth \\
\hline
\end{tabular}


Table 3 Significantly downregulated contigs (Continued)

\begin{tabular}{|c|c|c|c|c|}
\hline \multicolumn{5}{|l|}{ 7. Immunity } \\
\hline comp8292_c1_seq1 & Galactose-specific C-type lectin & 66.95 & 145.97 & Pathogen binding \\
\hline comp3093_c0_seq1 & $\begin{array}{l}\text { EGF-like module-containing mucin-like } \\
\text { hormone receptor-like } 1\end{array}$ & 2.09 & 11.49 & Immune signalling \\
\hline \multicolumn{5}{|l|}{ 8. Salivary proteins } \\
\hline comp6651_c0_seq1 & Short form d7 salivary protein sd7-1 & 39.94 & 79.53 & Salivary protein \\
\hline comp6296_c0_seq4 & Short form d7 salivary protein sd7-1 & 4.35 & 18.17 & Salivary protein \\
\hline \multicolumn{5}{|l|}{ 9. Mobile elements } \\
\hline comp14966_c0_seq1 & af377999_1 transposase & 1.08 & 6.80 & DNA integration \\
\hline comp334_c0_seq2 & line-1 retrotransposon-like & 0.00 & 6.43 & DNA integration \\
\hline comp4558_c0_seq2 & bel12_ag transposon polyprotein & 1.60 & 5.48 & Polyprotein \\
\hline comp13589_c0_seq12 & Uncharacterized protein K02A2.6-like & 1.25 & 5.23 & DNA integration \\
\hline comp4787_c0_seq1 & bel12_ag transposon polyprotein & 1.16 & 4.89 & Polyprotein \\
\hline
\end{tabular}

Contigs in italics are of bacterial origin. FPKM - Fragments Per Kilobase of transcript per Million mapped reads for the wFlu and Tet libraries

be utilised by Wolbachia to fabricate its membranous vacuoles. $w$ Flu infection also decreased levels of diacylglyceride (DAG) synthesis protein, suggesting that the infection likely interferes with DAG synthesis, and therefore lipid transport as DAGs are prominent transport lipids. Likewise, changes in the expression of these genes could potentially promote $P$. gallinaceum development.

Infection also induced expression of chorion peroxidase, an enzyme involved in ovarian follicle maturation, and decreased the levels of a vitellogenic carboxypeptidase, which is a yolk protein produced in the fat body [76]. The effect on these genes likely arises due to the strong presence of $w$ Flu in the ovaries, and may contribute to further differences in egg nutritional content, as is seen with glycogen [71].

\section{Membranes}

We observed that infection with $w$ Flu also affected 11 transcripts involved in membrane structure, or transmembrane transport. This included an ompa-like protein that is part of the Wolbachia membrane, which was indicative of Wolbachia replication. We also saw the upregulation of membrane proteins involved in the transport of glucose and cholesterol through niemann-pick type c2 protein, and these could potentially be involved in altering Plasmodium infection dynamics, given the varied metabolic requirements of the parasite [77]. This is further evidence of a co-opting of metabolic machinery, as Wolbachia has been strongly linked to carbohydrate metabolism as an energy source [38], and cholesterol, as a critical component of the vacuoles that surround the bacteria [78].

Infection also caused the upregulation of 3 membranebound chemosensory receptors potentially linked to Ae. fluviatilis behaviour. These three include sulfakinin, a digestive regulator in insects [79], and 2 taste receptors, including an ionotropic receptor and a gustatory receptor linked to sugar feeding [80]. Wolbachia infection can influence host olfaction at the behavioural $[81,82]$ and molecular level [11], and in the case of $w$ MelPop, also dramatically alters feeding behaviour [83]. These effects could possibly contribute to a minor change in olfactory response or potentially behaviour in response to $w \mathrm{Flu}$ infection. In contrast, only 3 membrane-related genes were differentially expressed in the Tet dataset, including an ATP-dependent transporter molecule of bacterial origin, and two membrane receptors with putative roles in immune signalling, and membrane-protein interactions.

\section{Physiology}

We saw that $w$ Flu upregulated several genes with a potential role in neurological function and development. These included calcium and sodium channels, a major allergen, a fasciculation and elongation protein, which is involved in nerve signal transduction, and neuroblast formation protein, and BMP-induced dendrite morphogenesis factor, which are both involved in neural development. While $w$ Flu does not heavily infect many non-reproductive tissues, it is present in the head at low density [15], and so could affect neurological function. Both $w \mathrm{Mel}$ and $w$ MelPop infect neural tissues $[15,17]$, and $w$ MelPop has a pronounced effect, causing neurological degradation in Drosophila melanogaster [84]. Wolbachia also affect hormone levels and contribute to host behavioural changes [85-87]. The overall physiological effect of $w$ Flu infection is unclear, however given that genes involved in neurological development, signalling and neurotransmitter trafficking 
and release were all upregulated by $w$ Flu, there could be critical effects.

While $w$ Flu does infect Ae. fluviatilis heads, it is not found in the omatidia cells of the eye, unlike wMelPop [15], yet $w$ Flu infection decreased the expression of 4 photoreceptor proteins involved in phototransduction, and 3 visual receptors, indicating that $w$ Flu may influence host visual perception. There have been no categorised effects of Wolbachia on host visual process, and no similar genes were affected by $w$ Mel or $w$ MelPop [11]. Potential physiological consequences of these changes could be a decreased sensitivity to light, which would be disadvantageous to the mosquito, or may indicate decreased activity during low level light conditions [88-90].

\section{Cellular processes}

There was also evidence that $w$ Flu infection altered common cellular processes by influencing the expression of genes involved in DNA repair and replication, and DNA packaging (Tables 2 and 3), with similar genes affected by other Wolbachia infection in mosquitoes particularly $w$ MelPop [11]. Other contigs were also linked to the processes of transcription and translation, and more specifically to mRNA processing, snRNA and rRNA processing, and protein folding, which is not unexpected given that Wolbachia produces and affects the production of small RNAs [91, 92].

We observed that $w$ Flu altered the expression of chromobox protein homolog 1 , a heterochromatin protein that could potentially be involved in epigenetic silencing of gene expression, or in chromosome integrity. Likewise, we observed altered levels of histone $2 b$, which is involved in DNA packaging as a key component of the nucleosome, and in gene silencing. Wolbachia infection in Ae. aegypti mosquitoes is known to affect cytosine methylation and gene silencing [93], and our results could indicate that there could potentially be a similar influence on silencing through histone modification. In that study, Wolbachia strongly altered the methylation profile of genes involved in membrane function including transport, and here we observed that a similar group of genes were affected during $w$ Flu infection in $A e$. fluviatilis.

Reproductive manipulation in Wolbachia-infected insects has also been associated with histones and chromatin. For instance, CI has been linked to delays in the deposition of histones in the male pronucleus [94], while male-killing has been linked to defective chromatin packaging, and altered chromosome behaviour [95]. Critically, the molecular effects of these processes are associated with adult males (CI), or in early stage embryos, and while our data were generated from adult females, these results may suggest that there are broader effects of Wolbachia infection on cellular processes related to DNA packaging, chromatin formation, and the regulation of gene expression in mosquitoes.

\section{Comparison to other Wolbachia infections in mosquitoes}

As $w$ Flu is a native Wolbachia infection, and has likely undergone a lengthy period of co-evolution with Ae. fluviatilis, we were quite surprised to discover that infection affected the expression of genes involved in a wide range of host biological processes. The scope of the transcriptional changes associated with infection (257 genes), appears to be similar to that of $w \mathrm{Mel}$ in Ae. aegypti (327 genes), although that study utilized a microarray rather than RNA-Seq, and the difference in sensitivity of the techniques may be a factor [11]. This similarity is interesting given that the latter association is both relatively novel, and is a transinfection that produces a broader range of physiological effects, and has greater bacterial density $[15,17,32]$. In contrast, transinfection of Ae. aegypti with the more virulent $w$ MelPop strain has been demonstrated to alter the expression of 244 genes in one study [14], and 2723 in another [11], with the studies utilizing different methods of analysis. A larger set of differentially expressed genes would likely reflect the pathogenicity associated with $w$ MelPop infection.

While these $w \mathrm{Mel}$ and $w$ MelPop data come from transinfections in a different species, we sought to determine if there was a core set of genes or functional groups of genes that were differentially expressed in all three associations, as this might explain something fundamental about the nature of Wolbachia infections in mosquitoes. 75 of the upregulated contigs, and 54 of the downregulated contigs were homologous to previously described Ae. aegypti transcripts in VectorBase. However, only 42 of these were also differentially expressed during either $w$ Mel or $w$ MelPop infection. Interestingly, the majority of these were upregulated by $w \mathrm{Mel}$ and $w$ MelPop, even if they were downregulated by $w$ Flu (Additional file 5), with this difference likely due to the relative novelty of the former transinfections.

Taking a broader approach, we then compared types of genes affected by all three strains, for example looking at all serine proteases, rather than a specific serine protease. Forty-six of the same types of genes were affected by both $w$ MelPop and $w$ Flu, with 33 upregulated and 13 downregulated (Additional file 5). For $w \mathrm{Mel}$ and $w \mathrm{Flu}$, 15 genes of the same type were affected, with 12 upregulated and 2 downregulated, all of which were also affected by $w$ MelPop (Table 4). Genes that are upregulated in the presence of Wolbachia reflect 5 key areas, protein and fat metabolism, redox process, membrane transport, DNA/RNA processing, and bacterial recognition, all of which have been previously characterised in Wolbachia infections $[10,11,38,72,96]$. Genes that were downregulated include cuticle proteins 
Table 4 Core genes affected by Wolbachia infection in mosquitoes

\begin{tabular}{ll}
\hline Upregulated & Downregulated \\
\hline Cytochrome p450 & Carboxypeptidase \\
Galactose-specific C-type lectin & Cuticle protein \\
Gram negative binding protein & \\
Membrane transport protein & \\
Odorant binding protein & \\
Pancreatic triacylglycerol lipase & \\
Permease & \\
Serine protease & \\
Serine/Threonine protein kinase & \\
Short-chain dehydrogenase & \\
Trypsin &
\end{tabular}

and carboxypeptidases, which are involved in protein digestion. Cuticle proteins can be downregulated in response to tetracycline treatment in Wolbachia-infected Brugia malayi worms [97]. They can also be downregulated in response to viral infections, and potentially play a role in host resistance to infection [98]. These processes likely contribute to making the mosquito host environment more favourable for Wolbachia propagation, and thus represent areas of interest for further study.

\section{Conclusions}

Here, we present the transcriptome of the mosquito Ae. fluviatilis, and consider the transcriptomic effects of its native Wolbachia strain, wFlu. Previous results suggest that $w$ Flu infects host tissues at relatively low densities, causes incomplete $\mathrm{CI}$ and has no observable fitness cost $[15,32]$, in accordance with theories that suggest native Wolbachia strains have lost bacterial density and pathogenicity during long periods of co-evolution, and the development of tolerance on the part of their hosts [26]. Our data indicated that $w$ Flu infection led to the differential expression of 257 genes, and while the scale of these changes was not as extreme as what is sometimes seen with Wolbachia transinfections in Ae. aegypti [10, $11,14]$, the effect was still broad in scope and encompassed a wide range of biological processes, many of which are held in common with Wolbachia infections in other mosquitoes. Metabolic effects of $w$ Flu infection appear to be particularly prominent [71], especially those associated with protein and lipid metabolism, and it is possible that the strain maintains the characteristic of a Jekyll and Hyde infection by both parasitising and providing key metabolites [26, 99]. And our results suggest that native strains such as $w$ Flu likely have a greater impact on mosquito host biology than previously thought.
Critically, we did not see evidence that $w$ Flu infection activated or suppressed the immune pathways typically associated with Wolbachia or Plasmodium infection $[100,101]$. However, we did observe changes to a range of genes involved in immunity, oxidative stress and metabolism that have previously been associated with Plasmodium infection $[59,102]$, and could feasibly play a role in the enhancement of $P$. gallinaceum. Further molecular changes contributing to enhancement could be induced by infection or blood feeding, or under certain conditions associated with infections in the vertebrate host, and these could explain why enhancement of P. gallinaceum does not occur consistently [32]. Plasmodium enhancement is more prominent amongst transient artificial Wolbachia infections, where there is typically more extreme manipulation of host immunity and bacterial density that could contribute to the phenotype [103], and where temperature appears to be a key determinant [30]. While transient infections will likely never be utilised outside of the laboratory, determining the prevalence of enhancement amongst mosquito vectors naturally infected by Wolbachia, and identifying the causal mechanism of enhancement remain important issues going forward.

\section{Methods}

\section{Mosquito material}

Two mosquito lines were used in these experiments. The original, Wolbachia-infected Aedes fluviatilis (wFlu) line was derived from mosquitoes originally captured in Belo Horizonte in 1975 [32]. The mosquito colony was maintained in the laboratory until 2013 when a subset (Tet) was treated with tetracycline hydrochloride to remove the native Wolbachia infection, and then had their gut microbiota recolonized, as previously described [32]. Colony larvae were reared at low density in dechlorinated water, and were fed with fish food (Goldfish Colour, Alcon, Camboriú, Santa Catarina, Cat. No. 0504-2). Adults were maintained in low-density cages in a climate-controlled insectary (temperature: $27 \pm 1{ }^{\circ} \mathrm{C}$, RH: $70 \pm 10 \%$, photoperiod: $12 \mathrm{~h}$ light: $12 \mathrm{~h}$ dark), and provided $10 \%$ sucrose solution ad libitum. Mosquitoes used in experiments were maintained in small cylindrical cages (diameter $-16 \mathrm{~cm}$, height $-18 \mathrm{~cm}$ ) of approximately 80-90 individuals. Experiments were conducted more than 2 years after tetracycline treatment. In all experiments, the Tet line served as a Wolbachiauninfected control line for the $w$ Flu line, to study the effects of Wolbachia infection.

\section{RNA extractions \& library preparation}

Six groups of samples were prepared for sequencing, with each group going on to form an independent library. Three groups each of 16 6-day old whole adult 
females from the Flu and Tet lines were collected and total RNA extracted using the $\mathrm{Trizol}^{\circ}$ protocol according to manufacturer's instructions (Invitrogen), for a total of 6 independent samples, with 3 biological replicates per treatment. Mosquitoes were fed only $10 \%$ sucrose prior to collection. RNA levels in each sample were quantified using a NanoDrop ND1000 (ThermoFisher Scientific). Sample degradation levels were checked by running a portion of the samples on a standard non-denaturing agarose gel containing bleach [104].

cDNA libraries were constructed using Illumina Truseq RNA Sample Preparation Kit (Illumina Inc.) according to the manufacturer instructions starting with $4 \mu \mathrm{g}$ of total RNA. The library products were then sequenced using an Illumina MiSeq platform on a paired-end $300 \mathrm{bp}$ run. After cleaning reads from adaptor sequences, the quality of the reads was assessed using the FastQC program (http:// www.bioinformatics.babraham.ac.uk/projects/fastqc/).

Cleaned reads are available for download at the National Center for Biotechnology Information - Sequence Read Archive under the BioProject ID PRJNA320882.

\section{De novo transcriptome assembly and contig annotation}

Since no reference genome was available for Aedes fluviatilis, a de novo transcriptome assembly was built with Trinity (https:/github.com/trinityrnaseq/trinityrnaseq/ wiki) using default parameters. All six Illumina RNAseq datasets were combined in order to assemble a more reliable transcriptome, with a total of 19,919,299 paired-end, high quality reads. Contig sequences were searched for candidate proteins with TransDecoder (https://transdecoder.github.io/), again using standard parameters. The assembled contigs were annotated through local alignments with BlastX (http://blast.ncbi.nlm.nih.gov/Blast.cgi) to the NCBI non-redundant (NR) and KEGG databases. BlastX parameters were set with an -e value of 1e-10. Blast2GO (https://www.blast2go.com/) was used to retrieve Gene Ontologies to annotated transcripts. Phylogenetic and divergence analyses were conducted using sequence data obtained during this study, or from UniProt (www.uniprot.org) or VectorBase (https://www.vectorbase.org). Methods and references for these analyses are described in Additional file 1.

\section{Read mapping and differential gene expression}

All Illumina paired-end reads libraries were mapped separately against the Ae. aegypti predicted transcriptome, available at VectorBase, and the Trinity assembled contigs, both with Bowtie2 (http://bowtie-bio.sourceforge.net/ bowtie2/index.shtml) using the default parameters while configuring fragment length. The Integrative Genomics Viewer (http://www.broadinstitute.org/igv/) was used to visualize the reads that were mapped back to the assembled transcriptome. Read counts mapped to each transcript were acquired with a custom Pearl script (available upon request).

Two R packages from Bioconductor (www.bioconductor.org), baySeq and DESeq2, were selected in order to identify the contigs that were significantly differentially expressed due to the presence of Wolbachia. The baySeq method is more sensitive, but also carries a greater false positive call rate, hence differential expression was confirmed using DESeq2 [45, 105]. The baySeq FDR (false discovery rate) $P$ value for multiple tests was set to 0.05 . The DESeq2 adjusted $P$ value was set to 0.01 , and transcripts were filtered according to their log2FoldChange (higher than +1 or lower than -1 ). We then obtained an estimate of the average fold change for each of the differentially expressed contigs using FPKM (fragments per kilobase of exon per million fragments mapped). Contigs that were differentially expressed but had no BlastX hit were excluded from analysis.

$\mathrm{GO}$ terms for the remaining contigs were generated through Blast2GO (https://www.blast2go.com) and VectorBase, or, when data could not be discovered from these sources, through FlyBase (http://flybase.org) or InterPro (http://www.ebi.ac.uk/interpro/), based on the analysis of homologous genes. GO information from contigs that matched to the same BlastX hit was considered only once, to avoid double counting. These GO lists were compiled and used to create two lists, one for the Flu libraries with the native wFlu Wolbachia infection, and the other for the Tet libraries, where the Wolbachia had been removed. Contigs were grouped according to putative function, and these lists were then compared to develop a profile of the transcriptomic effects of $w$ Flu on its mosquito host.

Differentially expressed contig functions and GOs were then compared against the transcriptomic data from $w \mathrm{Mel}$ and wMelPop-infected Aedes aegypti arrays [11, 14], in order to develop a profile of types of transcriptional changes held in common between the three Wolbachia strains and their mosquito hosts. Contigs that had BlastX matches against Ae. aegypti were compared directly with Ae. aegypti expression data. Those contigs that matched to another species were compared directly against the Ae. aegypti genome in NCBI using BlastN (http:// blast.ncbi.nlm.nih.gov/Blast.cgi). Any hits with a substantial match percentage $(>80 \%)$ and a significant e-value were used for further analysis, using the first hit in a comparison in VectorBase while those that did not were not considered. This information was used to determine if any of the specific genes affected by $w$ Flu infection were also affected by $w$ Mel or $w$ MelPop infection.

\section{Confirmation of differential expression}

To assess the accuracy of the transcriptomic data set, six contigs were selected at random for expression analysis 
with RT-qPCR. Two of these contigs were indicated to have higher expression for Flu mosquitoes (AF10645; comp10645_c1_seq2 and AF10453; comp10453_c0_seq5), two had higher expression in Tet mosquitoes (AF15178; comp15178_c0_seq1 and AF14155; comp14155_c0_seq1), while two had equivalent expression between Flu and Tet mosquitoes (AF2025; comp2025_c0_seq1 and AF2041; comp2041_c0_seq1). The first four of these contigs were predicted to be differentially expressed through both the bayseq and DESeq2 analyses. Primers for these contigs were designed from the sequences generated during sequencing, which meant that they were suitable for cDNA. Primer sequences were designed using Primer 3 V0.4.0 (http://bioinfo.ut.ee/primer3$0.4 .0 /$ ) to have a $\mathrm{Tm}$ of $55-60{ }^{\circ} \mathrm{C}$ and a product size range of $80-120 \mathrm{bp}$ (Additional file 6).

Mosquitoes from both the Flu and Tet lines were reared as described above, and then females were collected individually at 6 days post eclosion. Total RNA was extracted and quantified as described above. First strand cDNA synthesis was conducted with $1 \mu \mathrm{g}$ of total RNA from each sample using the M-MLV reverse transcriptase assay according to manufacturer's instructions (Promega cat: C118A). cDNAs were then diluted 1:10 in nuclease-free water and stored at $-30{ }^{\circ} \mathrm{C}$. SYBR-based PCR was used to confirm the expression of each of the test genes, with 15 samples tested per mosquito line. Each gene was quantified in duplicate for all samples using the following mix: SYBR $-5 \mu \mathrm{L}$, forward and reverse primers $(10 \mu \mathrm{M})-0.5 \mu \mathrm{L}$ each, sterile RNase free water - $2 \mu \mathrm{L}$, sample $2 \mu \mathrm{L}$ ). RT-qPCR for samples was run on a LightCycler 96 System (Roche) using the following profile: $10 \mathrm{~min}$ pre-incubation at $95^{\circ} \mathrm{C}, 40$ cycles of $15 \mathrm{~s}$ at $95^{\circ} \mathrm{C}, 60 \mathrm{~s}$ at $60^{\circ} \mathrm{C}$, melt curve $-95^{\circ} \mathrm{C}$ for $15 \mathrm{~s}$, ramp from $60{ }^{\circ} \mathrm{C}$ to $95{ }^{\circ} \mathrm{C}$ at $1.6{ }^{\circ} \mathrm{C} / \mathrm{s}$. Expression values for each gene were normalised against actin1 expression, which had previously been demonstrated to be a good control gene for Ae. fluviatilis [32]. Mean normalised expression values for each gene were calculated using Q-Gene [106], and were compared statistically between Flu and Tet mosquitoes using Mann Whiney $U$ tests (Prism v 6.0 g, Graphpad).

\section{Additional files}

Additional file 1: Divergence and phylogenetic analyses. Phylogenetic trees, and an estimate of the time of divergence for Aedes fluviatilis and other key mosquito species. (DOCX $1815 \mathrm{~kb}$ )

Additional file 2: Contigs of Wolbachia origin. List of contigs identified as being from Wolbachia pipientis. (XLSX 59 kb)

Additional file 3: Annotated, differentially expressed Ae. fluviatilis contigs. Table depicting all differentially expressed contigs, relevant annotations, and expression data. (XLSX $55 \mathrm{~kb}$ )

Additional file 4: $\mathrm{GO}$ terms with multiple hits associated with wFlu infection. Table depicting GO terms that were associated with more than one differentially expressed contig, for Wolbachia-infected and -uninfected mosquitoes. (DOCX $124 \mathrm{~kb}$ )

Additional file 5: Comparison of the transcriptomic effects of $w F l u$ with wMel and wMelPop infection in Ae. aegypti. Two tables. The first depicts gene homologs that are differentially expressed in wFlu-infected Aedes fluviatilis and/or wMel-infected Aedes aegypti, and wMelPop-infected Aedes aegypti. The second depicts gene functions that are commonly differentially expressed between the three associations. (DOCX $154 \mathrm{~kb}$ )

Additional file 6: List of primers used for RT-qPCR confirmation of differential expression. Oligonucleotide sequences for genes used in this work. (DOCX $63 \mathrm{~kb}$ )

\section{Abbreviations}

ATP: Adenine triphosphate; bp: Base pairs; Cl: Cytoplasmic incompatibility; DAG: Diacylglyceride; FPKM: Fragments per kilobase of transcript per million mapped reads; GO: Gene ontology; Kb: Kilobase; KEGG: Kyoto encyclopedia of genes and genomes; KO: Kegg orthology; NR: NCBI non-redundant database; RNA-Seq: RNA sequencing (whole transcriptome shotgun sequencing); ROS: Reactive oxygen species; rRNA: Ribosomal RNA; RT-qPCR: Quantitative reverse transcriptase $P C R$; snRNA: Small nuclear RNA

\section{Acknowledgements}

The authors wish to thank Dr Paulo Ribolla from UNESP for use of the Illumina MiSeq platform and the INCT-EM for the Real-Time PCR machine. We also wish to thank Alice Sabatino and Anna Paula Canuto for technical support. Finally, we thank Dr Victor Pylro and Dr Larissa Scholte from FlOCRUZ/Minas for assistance with the phylogenetic analyses.

\section{Funding}

This work was supported by FAPEMIG, CNPq, and CAPES. FSP received a CNPq post-doctoral fellowship (process number 168223/2014-7).

\section{Availability of data and materials}

All RNA sequencing data are available for download at the National Center for Biotechnology Information - Sequence Read Archive under the BioProject ID PRJNA320882.

\section{Authors' contributions}

EPC participated in study design, performed the RT-qPCR validation assay, carried out comparative data analysis, and drafted the manuscript. FSP performed the sequence assembly, annotation, analysis of differential expression, phylogenetic analysis, and helped to draft the manuscript. LAB conceived and designed the study, prepared samples for sequencing, conducted the divergence analysis, and helped prepare the manuscript. JBLS prepared samples and performed the RT-qPCR validation assay. MHFS helped to design the study, and prepared RNA libraries for sequencing. LAM conceived and designed the study, drafted the manuscript and supervised the research. All authors contributed to and approved the final version of the manuscript.

\section{Competing interests}

The authors declare that they have no competing interests.

Consent for publication

Not applicable.

Ethics approval and consent to participate

Not applicable.

\section{Author details}

${ }^{1}$ Grupo Mosquitos Vetores: Endossimbiontes e Interação Patógeno Vetor, Centro de Pesquisas René Rachou - Fiocruz, Belo Horizonte, Minas Gerais, Brazil. ${ }^{2}$ Grupo de Informática de Biossistemas e Genômica, Centro de Pesquisas René Rachou - Fiocruz, Belo Horizonte, Minas Gerais, Brazil. ${ }^{3}$ Instituto de Bioquímica Médica, Universidade Federal do Rio de Janeiro, Rio de Janeiro, Brazil.

Received: 24 August 2016 Accepted: 19 December 2016 Published online: 03 January 2017 


\section{References}

1. Zug R, Hammerstein P. Still a host of hosts for Wolbachia: analysis of recent data suggests that $40 \%$ of terrestrial arthropod species are infected. PLoS One. 2012;7(6), e38544.

2. Caragata EP, Dutra HL, Moreira LA: Exploiting intimate relationships: Controlling mosquito-transmitted disease with Wolbachia. Trends Parasitol. 2016

3. Werren JH, Baldo L, Clark ME. Wolbachia: master manipulators of invertebrate biology. Nat Rev Microbiol. 2008;6(10):741-51.

4. McGraw EA, O'Neill SL. Beyond insecticides: new thinking on an ancient problem. Nat Rev Microbiol. 2013;11(3):181-93.

5. Vector-borne diseases. WHO Fact sheets. World Health Organization; 2016.

6. Hoffmann AA, Montgomery BL, Popovici J, Iturbe-Ormaetxe I, Johnson PH, Muzzi F, Greenfield M, Durkan M, Leong YS, Dong Y, et al. Successful establishment of Wolbachia in Aedes populations to suppress dengue transmission. Nature. 2011;476(7361):454-U107.

7. Turelli M, Hoffmann AA. Rapid spread of an inherited incompatibility factor in California Drosophila. Nature. 1991;353(6343):440-2.

8. Z Zhang D, Lees RS, Xi Z, Gilles JR, Bourtzis K. Combining the Sterile Insect Technique with Wolbachia-Based Approaches: II-A Safer Approach to Aedes albopictus Population Suppression Programmes, Designed to Minimize the Consequences of Inadvertent Female Release. PLoS One. 2015;10(8), e0135194

9. Molloy JC, Sinkins SP. Wolbachia Do Not Induce Reactive Oxygen SpeciesDependent Immune Pathway Activation in Aedes albopictus. Viruses. 2015; 7(8):4624-39.

10. Pan X, Zhou G, Wu J, Bian G, Lu P, Raikhel AS, Xi Z. Wolbachia induces reactive oxygen species (ROS)-dependent activation of the Toll pathway to control dengue virus in the mosquito Aedes aegypti. Proc Natl Acad Sci U S A. 2012;109(1):E23-31.

11. Rancès $\mathrm{E}, \mathrm{Ye} \mathrm{YH}$, Woolfit M, McGraw EA, O'Neill SL. The relative importance of innate immune priming in Wolbachia-mediated Dengue interference. Plos Pathogens. 2012;8(2):e1002548.

12. Wong ZS, Brownlie JC, Johnson KN. Oxidative stress correlates with Wolbachia-mediated antiviral protection in Wolbachia-Drosophila associations. Appl Environ Microbiol. 2015;81(9):3001-5

13. Hussain M, Lu G, Torres S, Edmonds JH, Kay BH, Khromykh AA, Asgari S. Effect of Wolbachia on replication of West Nile virus in a mosquito cell line and adult mosquitoes. J Virol. 2013;87(2):851-8.

14. Kambris Z, Cook PE, Phuc HK, Sinkins SP. Immune activation by lifeshortening Wolbachia and reduced filarial competence in mosquitoes. Science. 2009;326(5949):134-6.

15. Moreira LA, Iturbe-Ormaetxe I, Jeffery JA, Lu GJ, Pyke AT, Hedges LM, Rocha BC, Hall-Mendelin S, Day A, Riegler M, et al. A Wolbachia symbiont in Aedes aegypti limits infection with Dengue, Chikungunya, and Plasmodium. Cell. 2009;139(7):1268-78

16. van den Hurk AF, Hall-Mendelin S, Pyke AT, Frentiu FD, McElroy K, Day A, Higgs S, O'Neill SL. Impact of Wolbachia on infection with chikungunya and yellow fever viruses in the mosquito vector Aedes aegypti. PLoS Negl Trop Dis. 2012:6(11), e1892.

17. Walker $T$, Johnson PH, Moreira LA, Iturbe-Ormaetxe I, Frentiu FD, McMeniman CJ, Leong YS, Dong Y, Axford J, Kriesner P, et al. A non-virulent Wolbachia infection blocks dengue transmission and rapidly invades Aedes aegypti populations. Nature. 2011;476:450-5

18. Ye YH, Woolfit M, Rances E, O'Neill SL, McGraw EA. Wolbachia-associated bacterial protection in the mosquito Aedes aegypti. PLoS Negl Trop Dis. 2013;7(8), e2362

19. Dutra HL, Rocha MN, Dias FB, Mansur SB, Caragata EP, Moreira LA Wolbachia Blocks Currently Circulating Zika Virus Isolates in Brazilian Aedes aegypti Mosquitoes. Cell Host Microbe. 2016;19(6):771-4.

20. Bian G, Joshi D, Dong Y, Lu P, Zhou G, Pan X, Xu Y, Dimopoulos G, Xi Z. Wolbachia invades Anopheles stephensi populations and induces refractoriness to Plasmodium infection. Science. 2013;340(6133):748-51.

21. Ferguson NM, Kien DT, Clapham H, Aguas R, Trung VT, Chau TN, Popovici J, Ryan PA, O'Neill SL, McGraw EA, et al. Modeling the impact on virus transmission of Wolbachia-mediated blocking of dengue virus infection of Aedes aegypti. Sci Transl Med. 2015;7(279):279ra237.

22. Frentiu FD, Zakir T, Walker T, Popovici J, Pyke AT, van den Hurk A, McGraw EA, O'Neill SL. Limited dengue virus replication in field-collected Aedes aegypti mosquitoes infected with Wolbachia. PLoS Negl Trop Dis. 2014;8(2), e2688.

23. Hoffmann AA, Iturbe-Ormaetxe I, Callahan AG, Phillips BL, Billington $K$ Axford JK, Montgomery B, Turley AP, O'Neill SL. Stability of the WMel
Wolbachia Infection following invasion into Aedes aegypti populations. PLoS Negl Trop Dis. 2014;8(9), e3115

24. McMeniman CJ, Lane RV, Cass BN, Fong AWC, Sidhu M, Wang YF, O'Neill SL. Stable introduction of a life-shortening Wolbachia infection into the mosquito Aedes aegypti. Science. 2009;323(5910):141-4.

25. Xi Z, Khoo CC, Dobson SL. Wolbachia establishment and invasion in an Aedes aegypti laboratory population. Science. 2005;310(5746):326-8.

26. Zug R, Hammerstein P. Bad guys turned nice? A critical assessment of Wolbachia mutualisms in arthropod hosts. Biol Rev Camb Philos Soc. 2014; 90(1):89-111.

27. Zele F, Nicot A, Berthomieu A, Weill M, Duron O, Rivero A. Wolbachia increases susceptibility to Plasmodium infection in a natural system. Proc Biol Sci. 2014:281(1779):20132837.

28. Dodson BL, Hughes GL, Paul O, Matacchiero AC, Kramer LD, Rasgon JL. Wolbachia enhances West Nile virus (WNV) infection in the mosquito Culex tarsalis. PLoS Negl Trop Dis. 2014;8(7), e2965.

29. Hughes $G L$, Vega-Rodriguez J, Xue P, Rasgon JL. Wolbachia strain wAlbB enhances infection by the rodent malaria parasite Plasmodium berghei in Anopheles gambiae mosquitoes. Appl Environ Microbiol. 2012;78(5):1491-5.

30. Murdock CC, Blanford S, Hughes GL, Rasgon JL, Thomas MB. Temperature alters Plasmodium blocking by Wolbachia. Sci Rep. 2014:4:3932.

31. Tason de Camargo M, Krettli AU. Aedes fluviatilis (Lutz), a new experimental host for Plasmodium gallinaceum brumpt. J Parasitol. 1978;64(5):924-5.

32. Baton LA, Pacidonio EC, Goncalves DS, Moreira LA. wFlu: characterization and evaluation of a native Wolbachia from the mosquito Aedes fluviatilis as a potential vector control agent. PLoS One. 2013;8(3), e59619.

33. Multini LC, Marrelli MT, Wilke AB. Microsatellite loci cross-species transferability in Aedes fluviatilis (Diptera:Culicidae): a cost-effective approach for population genetics studies. Parasit Vectors. 2015;8:635.

34. Nene V, Wortman JR, Lawson D, Haas B, Kodira C, Tu ZJ, Loftus B, Xi Z, Megy K, Grabherr M, et al. Genome sequence of Aedes aegypti, a major arbovirus vector. Science. 2007:316(5832):1718-23.

35. Duplouy A, Iturbe-Ormaetxe I, Beatson SA, Szubert JM, Brownlie JC, McMeniman CJ, McGraw EA, Hurst GD, Charlat S, O'Neill SL, et al. Draft genome sequence of the male-killing Wolbachia strain wBol1 reveals recent horizontal gene transfers from diverse sources. BMC Genomics. 2013:14:20.

36. Gill AC, Darby AC, Makepeace BL. Iron necessity: the secret of Wolbachia's success? PLoS Negl Trop Dis. 2014:8(10), e3224.

37. Zug R, Hammerstein P. Wolbachia and the insect immune system: what reactive oxygen species can tell us about the mechanisms of Wolbachiahost interactions. Front Microbiol. 2015;6:1201

38. Wu M, Sun LV, Vamathevan J, Riegler M, Deboy R, Brownlie JC, McGraw EA, Martin W, Esser C, Ahmadinejad N, et al. Phylogenomics of the reproductive parasite Wolbachia pipientis WMel: A streamlined genome overrun by mobile genetic elements. Plos Biol. 2004;2(3):327-41.

39. Iturbe-Ormaetxe I, Burke GR, Riegler M, O'Neill SL. Distribution, expression, and motif variability of ankyrin domain genes in Wolbachia pipientis. Bacteriol. 2005;187(15):5136-45.

40. Foster J, Ganatra M, Kamal I, Ware J, Makarova K, Ivanova N, Bhattacharyya A, Kapatral V, Kumar S, Posfai J, et al. The Wolbachia genome of Brugia malayi: endosymbiont evolution within a human pathogenic nematode. Plos Biol. 2005;3(4), e121

41. Sutton ER, Harris SR, Parkhill J, Sinkins SP. Comparative genome analysis of Wolbachia strain wAu. BMC Genomics. 2014;15:928.

42. Woolfit M, Iturbe-Ormaetxe I, Brownlie JC, Walker T, Riegler M, Seleznev A Popovici J, Rances E, Wee BA, Pavlides J, et al. Genomic evolution of the pathogenic Wolbachia strain, wMelPop. Genome Biol Evol. 2013;5(11):2189-204.

43. Duron O, Boureux A, Echaubard P, Berthomieu A, Berticat C, Fort P, Weill M. Variability and expression of ankyrin domain genes in Wolbachia variants infecting the mosquito Culex pipiens. J Bacteriol. 2007;189(12):4442-8.

44. Holt RA, Subramanian GM, Halpern A, Sutton GG, Charlab R, Nusskern DR, Wincker P, Clark AG, Ribeiro JM, Wides R, et al. The genome sequence of the malaria mosquito Anopheles gambiae. Science. 2002;298(5591):129-49.

45. Kvam VM, Liu P, Si Y. A comparison of statistical methods for detecting differentially expressed genes from RNA-seq data. Am J Bot. 2012;99(2):248-56.

46. Bourtzis K, Pettigrew MM, O'Neill SL. Wolbachia neither induces nor suppresses transcripts encoding antimicrobial peptides. Insect Mol Biol. 2000;9(6):635-9.

47. Chrostek E, Marialva MS, Yamada R, O'Neill SL, Teixeira L. High anti-viral protection without immune upregulation after interspecies Wolbachia transfer. PLoS One. 2014;9(6), e99025 
48. Warr E, Das S, Dong Y, Dimopoulos G. The Gram-negative bacteria-binding protein gene family: its role in the innate immune system of Anopheles gambiae and in anti-Plasmodium defence. Insect Mol Biol. 2008;17(1):39-51.

49. Osta MA, Christophides GK, Kafatos FC. Effects of mosquito genes on Plasmodium development. Science. 2004;303(5666):2030-2.

50. Berois M, Romero-Severson J, Severson DW. RNAi knock-downs support roles for the mucin-like (AeIMUC1) gene and short-chain dehydrogenase/ reductase (SDR) gene in Aedes aegypti susceptibility to Plasmodium gallinaceum. Med Vet Entomol. 2012;26(1):112-5.

51. Groat-Carmona AM, Kain H, Brownell J, Douglass AN, Aly AS, Kappe SH. A Plasmodium a/ $\beta$-hydrolase modulates the development of invasive stages. Cell Microbiol. 2015;17(12):1848-67.

52. Lamour SD, Straschil U, Saric J, Delves MJ. Changes in metabolic phenotypes of Plasmodium falciparum in vitro cultures during gametocyte development. Malar J. 2014;13:468.

53. Salcedo-Sora JE, Caamano-Gutierrez E, Ward SA, Biagini GA. The proliferating cell hypothesis: a metabolic framework for Plasmodium growth and development. Trends Parasitol. 2014;30(4):170-5.

54. Koo IC, Ohol YM, Wu P, Morisaki JH, Cox JS, Brown EJ. Role for lysosomal enzyme beta-hexosaminidase in the control of mycobacteria infection. Proc Natl Acad Sci U S A. 2008;105(2):710-5.

55. Rodrigues J, Oliveira GA, Kotsyfakis M, Dixit R, Molina-Cruz A, Jochim R, Barillas-Mury C. An epithelial serine protease, AgESP, is required for Plasmodium invasion in the mosquito Anopheles gambiae. PLoS One. 2012; 7(4), e35210.

56. Sharma S, Jadli M, Singh A, Arora K, Malhotra P. A secretory multifunctional serine protease, DegP of Plasmodium falciparum, plays an important role in thermo-oxidative stress, parasite growth and development. FEBS J. 2014; 281(6):1679-99.

57. Brennan LJ, Haukedal JA, Earle JC, Keddie B, Harris HL. Disruption of redox homeostasis leads to oxidative DNA damage in spermatocytes of Wolbachia-infected Drosophila simulans. Insect Mol Biol. 2012;21(5):510-20.

58. Zhang $Y K$, Ding $X L$, Rong $X$, Hong $X Y$. How do hosts react to endosymbionts? A new insight into the molecular mechanisms underlying the Wolbachia-host association. Insect Mol Biol. 2015;24(1):1-12.

59. Molina-Cruz A, DeJong RJ, Charles B, Gupta L, Kumar S, Jaramillo-Gutierrez G, Barillas-Mury C. Reactive oxygen species modulate Anopheles gambiae immunity against bacteria and Plasmodium. J Biol Chem. 2008:283(6):3217-23.

60. Esterhazy D, King MS, Yakovlev G, Hirst J. Production of reactive oxygen species by complex I (NADH:ubiquinone oxidoreductase) from Escherichia coli and comparison to the enzyme from mitochondria. Biochemistry. 2008; 47(12):3964-71.

61. Raha $\mathrm{S}$, Robinson $\mathrm{BH}$. Mitochondria, oxygen free radicals, disease and ageing. Trends Biochem Sci. 2000;25(10):502-8.

62. Brownlie JC, Cass BN, Riegler M, Witsenburg JJ, Iturbe-Ormaetxe I, McGraw EA, O'Neill SL. Evidence for metabolic provisioning by a common invertebrate endosymbiont, Wolbachia pipientis, during periods of nutritional stress. PLoS Pathog. 2009;5(4), e1000368.

63. Kosmidis S, Missirlis F, Botella JA, Schneuwly S, Rouault TA, Skoulakis EM. Behavioral decline and premature lethality upon pan-neuronal ferritin overexpression in Drosophila infected with a virulent form of Wolbachia. Front Pharmacol. 2014;5:66.

64. Kremer N, Voronin DA, Charif D, Mavingui P, Mollereau B, Vavre F. Wolbachia interferes with ferritin expression and iron metabolism in insects. Plos Pathogens. 2009;5(10), e1000630.

65. Ke H, Sigala PA, Miura K, Morrisey JM, Mather MW, Crowley JR, Henderson JP, Goldberg DE, Long CA, Vaidya AB. The heme biosynthesis pathway is essential for Plasmodium falciparum development in mosquito stage but not in blood stages. J Biol Chem. 2014;289(50):34827-37.

66. Ballard JW, Melvin RG. Tetracycline treatment influences mitochondrial metabolism and mtDNA density two generations after treatment in Drosophila. Insect Mol Biol. 2007;16(6):799-802.

67. Moriyama M, Nikoh N, Hosokawa T, Fukatsu T. Riboflavin Provisioning Underlies Wolbachia's Fitness Contribution to Its Insect Host. MBio. 2015; 6(6):e01732-01715.

68. Caragata EP, Rances E, Hedges LM, Gofton AW, Johnson KN, O'Neill SL, McGraw EA. Dietary cholesterol modulates pathogen blocking by Wolbachia. Plos Pathogens. 2013;9(6), e1003459.

69. Caragata EP, Rances E, O'Neill SL, McGraw EA. Competition for amino acids between Wolbachia and the mosquito host, Aedes aegypti. Microb Ecol. 2014;67(1):205-18.
70. Atyame CM, Labbe P, Lebon C, Weill M, Moretti R, Marini F, Gouagna LC, Calvitti M, Tortosa P. Comparison of Irradiation and Wolbachia Based Approaches for Sterile-Male Strategies Targeting Aedes albopictus. PLoS One. 2016:11(1), e0146834.

71. da Rocha FM, Martins R, Pessoa Costa E, Pacidonio EC, Araujo de Abreu L, da Silva Vaz Jr I, Moreira LA, da Fonseca RN, Logullo C. The modulation of the symbiont/host interaction between Wolbachia pipientis and Aedes fluviatilis embryos by glycogen metabolism. PLoS One. 2014;9(6), e98966.

72. Molloy JC, Sommer U, Viant MR, Sinkins SP. Wolbachia modulates lipid metabolism in Aedes albopictus mosquito cells. Appl Environ Microbiol. 2016:82(10):3109-20.

73. Vollmer J, Schiefer A, Schneider T, Julicher K, Johnston KL, Taylor MJ, Sahl HG, Hoerauf A, Pfarr K. Requirement of lipid II biosynthesis for cell division in cell wall-less Wolbachia, endobacteria of arthropods and filarial nematodes. Int J Med Microbiol. 2013;303(3):140-9.

74. Herren JK, Paredes JC, Schupfer F, Arafah K, Bulet P, Lemaitre B. Insect endosymbiont proliferation is limited by lipid availability. Elife. 2014;3, e02964

75. Atella GC, Bittencourt-Cunha PR, Nunes RD, Shahabuddin M, Silva-Neto MA. The major insect lipoprotein is a lipid source to mosquito stages of malaria parasite. Acta Trop. 2009;109(2):159-62.

76. Deitsch KW, Raikhel AS. Cloning and analysis of the locus for mosquito vitellogenic carboxypeptidase. Insect Mol Biol. 1993;2(4):205-13.

77. Labaied M, Jayabalasingham B, Bano N, Cha SJ, Sandoval J, Guan G, Coppens I. Plasmodium salvages cholesterol internalized by LDL and synthesized de novo in the liver. Cell Microbiol. 2011;13(4):569-86.

78. Cho KO, Kim GW, Lee OK. Wolbachia bacteria reside in host Golgi-related vesicles whose position is regulated by polarity proteins. PLoS One. 2011;6(7), e22703.

79. Zels S, Dillen S, Crabbe K, Spit J, Nachman RJ, Vanden Broeck J. Sulfakinin is an important regulator of digestive processes in the migratory locust, Locusta migratoria. Insect Biochem Mol Biol. 2015;61:8-16.

80. Arensburger P, Megy K, Waterhouse RM, Abrudan J, Amedeo P, Antelo B, Bartholomay L, Bidwell S, Caler E, Camara F, et al. Sequencing of Culex quinquefasciatus establishes a platform for mosquito comparative genomics. Science. 2010;330(6000):86-8.

81. Caragata EP, Real KM, Zalucki MP, McGraw EA. Wolbachia infection increases recapture rate of field-released Drosophila melanogaster. Symbiosis. 2011; 54(1):55-60.

82. Peng $Y$, Nielsen JE, Cunningham JP, McGraw EA. Wolbachia infection alters olfactory-cued locomotion in Drosophila spp. Appl Environ Microbiol. 2008; 74(13):3943-8.

83. Turley AP, Moreira LA, O'Neill SL, McGraw EA. Wolbachia infection reduces blood-feeding success in the Dengue fever mosquito. Aedes aegypti Plos Neglect Trop D. 2009;3(9), e516.

84. Strunov A, Kiseleva E, Gottlieb Y. Spatial and temporal distribution of pathogenic Wolbachia strain wMelPop in Drosophila melanogaster central nervous system under different temperature conditions. J Invertebr Pathol. 2013:114(1):22-30.

85. Liu C, Wang JL, Zheng Y, Xiong EJ, Li JJ, Yuan LL, Yu XQ, Wang YF. Wolbachia-induced paternal defect in Drosophila is likely by interaction with the juvenile hormone pathway. Insect Biochem Mol Biol. 2014;49:49-58.

86. Moreira LA, Ye YH, Turner K, Eyles DW, McGraw EA, O'Neill SL. The wMelPop strain of Wolbachia interferes with dopamine levels in Aedes aegypti. Parasit Vectors. 2011:4:28.

87. Rohrscheib CE, Bondy E, Josh P, Riegler M, Eyles D, van Swinderen B, Weible 2nd MW, Brownlie JC. Wolbachia Influences the Production of Octopamine and Affects Drosophila Male Aggression. Appl Environ Microbiol. 2015;81(14):4573-80

88. Jenkins AM, Muskavitch MA. Crepuscular Behavioral Variation and Profiling of Opsin Genes in Anopheles gambiae and Anopheles stephensi (Diptera: Culicidae). J Med Entomol. 2015;52(3):296-307.

89. Moon YM, Metoxen AJ, Leming MT, Whaley MA, O'Tousa JE. Rhodopsin management during the light-dark cycle of Anopheles gambiae mosquitoes. J Insect Physiol. 2014;70:88-93.

90. Rund SS, Gentile JE, Duffield GE. Extensive circadian and light regulation of the transcriptome in the malaria mosquito Anopheles gambiae. BMC Genomics. 2013;14:218.

91. Hussain M, O'Neill SL, Asgari S. Wolbachia interferes with the intracellular distribution of Argonaute 1 in the dengue vector Aedes aegypti by manipulating the host microRNAs. RNA Biol. 2013;10(12):1868-75.

92. Woolfit M, Algama M, Keith JM, McGraw EA, Popovici J. Discovery of putative small non-coding RNAs from the obligate intracellular bacterium Wolbachia pipientis. PLoS One. 2015;10(3), e0118595. 
93. Ye YH, Woolfit M, Huttley GA, Rances E, Caragata EP, Popovici J, O'Neill SL, McGraw EA. Infection with a virulent strain of Wolbachia disrupts genomewide patterns of cytosine methylation in the mosquito Aedes aegypti. PLoS One. 2013;8(6), e66482.

94. Landmann F, Orsi GA, Loppin B, Sullivan W. Wolbachia-mediated cytoplasmic incompatibility is associated with impaired histone deposition in the male pronucleus. PLoS Pathog. 2009;5(3), e1000343.

95. Riparbelli MG, Giordano R, Ueyama M, Callaini G. Wolbachia-mediated male killing is associated with defective chromatin remodeling. PLoS One. 2012;7(1), e30045.

96. Ikeya T, Broughton S, Alic N, Grandison R, Partridge L. The endosymbiont Wolbachia increases insulin/IGF-like signalling in Drosophila. Proc Biol Sci. 2009;276(1674):3799-807.

97. Ghedin E, Hailemariam T, DePasse JV, Zhang X, Oksov Y, Unnasch TR, Lustigman S. Brugia malayi gene expression in response to the targeting of the Wolbachia endosymbiont by tetracycline treatment. PLoS Negl Trop Dis. 2009;3(10), e525.

98. Colpitts TM, Cox J, Vanlandingham DL, Feitosa FM, Cheng G, Kurscheid S, Wang P, Krishnan MN, Higgs S, Fikrig E. Alterations in the Aedes aegypti transcriptome during infection with West Nile, dengue and yellow fever viruses. PLoS Pathog. 2011;7(9), e1002189.

99. Jiggins FM, Hurst GD. Rapid insect evolution by symbiont transfer. Science. 2011;332(6026):185-6.

100. Bahia AC, Kubota MS, Tempone AJ, Araujo HR, Guedes BA, Orfano AS, Tadei WP, Rios-Velasquez CM, Han YS, Secundino NF, et al. The JAK-STAT pathway controls Plasmodium vivax load in early stages of Anopheles aquasalis infection. PLoS Negl Trop Dis. 2011;5(11), e1317.

101. Garver LS, Dong Y, Dimopoulos G. Caspar controls resistance to Plasmodium falciparum in diverse anopheline species. PLoS Pathog. 2009;5(3), e1000335.

102. Liu K, Dong Y, Huang Y, Rasgon JL, Agre P. Impact of trehalose transporter knockdown on Anopheles gambiae stress adaptation and susceptibility to Plasmodium falciparum infection. Proc Natl Acad Sci U S A. 2013;110(43):17504-9.

103. Hughes GL, Koga R, Xue P, Fukatsu T, Rasgon JL. Wolbachia infections are virulent and inhibit the human malaria parasite Plasmodium falciparum in Anopheles gambiae. PLoS Pathog. 2011;7(5), e1002043.

104. Aranda PS, LaJoie DM, Jorcyk CL. Bleach gel: a simple agarose gel for analyzing RNA quality. Electrophoresis. 2012;33(2):366-9.

105. Xu X, Zhang Y, Williams J, Antoniou E, McCombie WR, Wu S, Zhu W, Davidson NO, Denoya P, Li E. Parallel comparison of Illumina RNA-Seq and Affymetrix microarray platforms on transcriptomic profiles generated from 5-aza-deoxy-cytidine treated HT-29 colon cancer cells and simulated datasets. BMC Bioinformatics. 2013;14 Suppl 9:S1.

106. Simon P. Q-Gene: processing quantitative real-time RT-PCR data. Bioinformatics. 2003;19(11):1439-40

\section{Submit your next manuscript to BioMed Central and we will help you at every step:}

- We accept pre-submission inquiries

- Our selector tool helps you to find the most relevant journal

- We provide round the clock customer support

- Convenient online submission

- Thorough peer review

- Inclusion in PubMed and all major indexing services

- Maximum visibility for your research

Submit your manuscript at www.biomedcentral.com/submit

) Biomed Central 